A obstrução de Euler de uma função 
SERVIÇO DE PÓS-GRADUAÇÃO DO ICMC-USP

Data de Depósito:

Assinatura:

\title{
A obstrução de Euler de uma função
}

\author{
Daiane Alice Henrique
}

Orientador: Prof. Dr. Nivaldo de Góes Grulha Júnior

Dissertação apresentada ao Instituto de Ciências Matemáticas e de Computação - ICMC-USP, como parte dos requisitos para obtenção do título de Mestre em Ciências - Matemática . VERSÃO REVISADA 
Ficha catalográfica elaborada pela Biblioteca Prof. Achille Bassi e Seção Técnica de Informática, ICMC/USP, com os dados fornecidos pelo(a) autor(a)

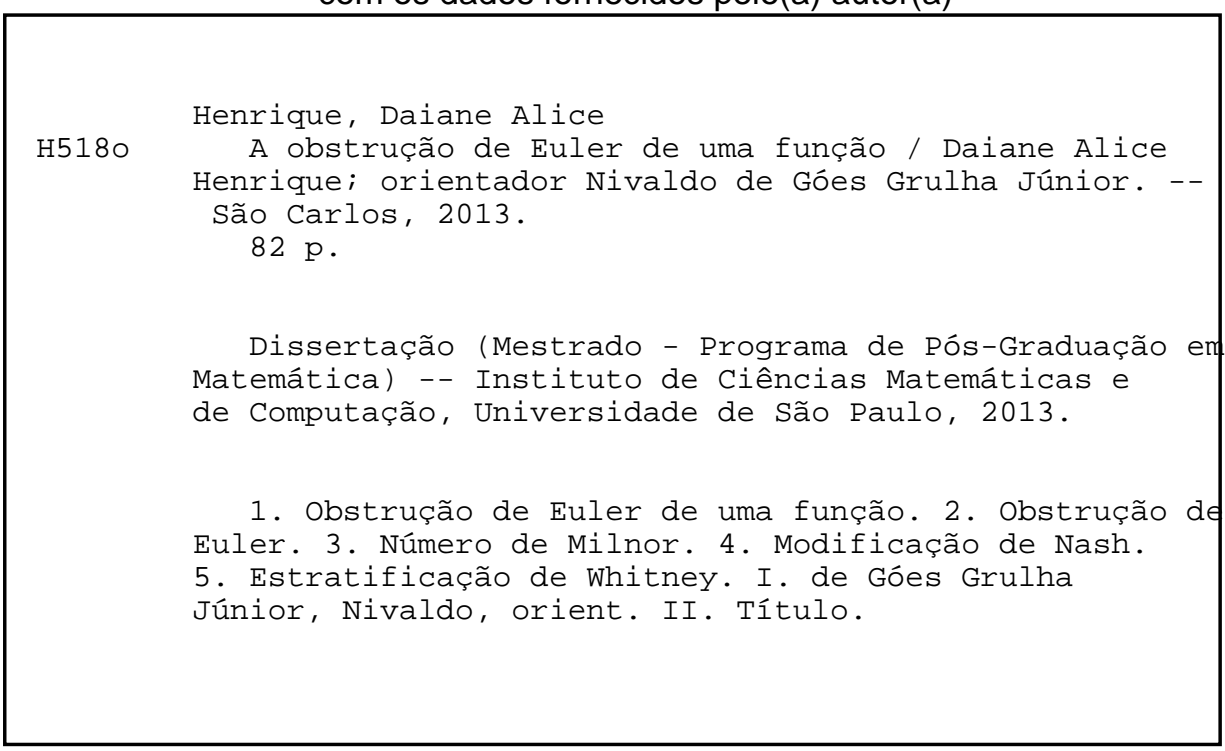


Aos meus pais José e Natalicia.

Aos meus irmãos Débora e Deivid.

E ao meu amado Andrews. 

"Não há ramo da Matemática, por mais abstrato que seja, que não possa um dia vir a ser aplicado aos fenômenos do mundo real".

Lobachevsky 



\section{Agradecimentos}

Sem o apoio e o incentivo das pessoas que amo, não poderia de modo algum realizar este sonho, agradeço a todos vocês que me auxiliaram nessa caminhada, em especial...

Agradeço a Deus, pela minha vida, pelas forças renovadas todos os dias e por mais esse sonho realizado.

Aos meus pais, por todos os ensinamentos, por todo amor e por toda a dedicação.

Aos meus irmãos, por todo apoio, pela compreensão e carinho.

Ao meu noivo Andrews, por todo o incentivo e amor, obrigada por acreditar em mim mais do que eu mesma.

Às amigas Amanda e Carol, pela companhia nos estudos e pela grande amizade.

Às amigas Camila e Thaís, pela disponibilidade e por toda ajuda.

Ao meu orientador Nivaldo, pela confiança e dedicação. Por sua paciência e pelos grandes ensinamentos.

À professora Eliris, pela amizade, por todo apoio e por sempre ter me incentivado a lutar por esse sonho.

Aos professores e funcionários do departamento de Matemática da UNESP/Rio Claro e do Instituto de Ciências Matemáticas e de Computação da USP/São Carlos.

Aos amigos que conheci durante minha graduação na Unesp/Rio Claro e durante o mestrado na USP/São Carlos. Muito obrigada!

À Fapesp pelo apoio financeiro para realização deste trabalho. 



\section{Resumo}

Nosso objetivo neste trabalho é estudar a obstrução de Euler de uma função, este conceito foi definido por J.-P. Brasselet, D. Massey, A. J. Parameswaran e J. Seade, e generaliza dois conceitos importantes, a obstrução de Euler definida por R. D. MacPherson assim como o número de Milnor de uma função.

O resultado principal deste trabalho mostra a relação existente entre a obstrução de Euler e a obstrução de Euler de uma função. 



\section{Abstract}

Our goal in this work is to study the Euler obstruction of a function, this concept was defined by J.-P. Brasselet, D. Massey, A. J. Parameswaran and J. Seade, and it generalizes two important concepts, the Euler obstruction defined by R. D. MacPherson and the Milnor number of a function.

The main result of this study shows the relation between the Euler obstruction and the Euler obstruction of a function. 



\section{Sumário}

$\begin{array}{ll}\text { Introdução } & 15\end{array}$

\begin{tabular}{lll}
\hline & Preliminares & 17
\end{tabular}

1.1 Variedades diferenciáveis . . . . . . . . . . . . . . . . . . . . . . . 17

1.2 Homotopia . . . . . . . . . . . . . . . . . . . . . . . . . 30

1.3 Homologia singular . . . . . . . . . . . . . . . . . . . . . . . 32

1.4 Cohomologia $\ldots \ldots \ldots \ldots \ldots \ldots \ldots$

1.5 Teoria de singularidades . . . . . . . . . . . . . . . . . . . . . . 44

1.6 Variedades algébricas e espaços analíticos complexos . . . . . . . . . . . . . 45

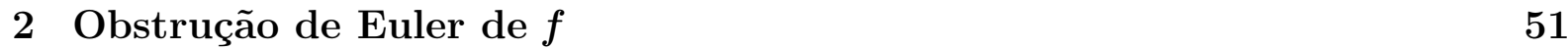

2.1 Estratificação de Whitney . . . . . . . . . . . . . . . . . . . . . . 51

2.2 Modificação de Nash . . . . . . . . . . . . . . . . . . . . . . . . . . . 54

2.3 Obstrução de Euler . . . . . . . . . . . . . . . . . . . . . . . . . . . . 56

2.4 Uma Fórmula Topológica . . . . . . . . . . . . . . . . . . . . . . . . . . . . 59

2.5 Obstrução de Euler de $f$. . . . . . . . . . . . . . . . . . . . . . . 60

\begin{tabular}{lll}
\hline 3 & Teorema Principal & 67
\end{tabular}

3.1 Teorema Principal $\ldots \ldots \ldots$. . . . . . . . . . . . . . . . . . . . 67

3.2 Lemas . . . . . . . . . . . . . . . . . . . . . . 73

3.3 Demonstração do Lema $\mid 3.1 .2 \ldots \ldots \ldots$. . . . . . . . . . . . . . . 77

\begin{tabular}{lll}
\hline 4 Conclusão & 79
\end{tabular} 



\section{Introdução}

A obstrução local de Euler, ou simplesmente obstrução de Euler, foi introduzida por R. D. MacPherson em [16] como uma de suas principais ferramentas na demonstração da conjectura de Deligne e Grothendieck sobre a existência e unicidade de classes características de variedades singulares. Uma definição equivalente deste conceito foi dada por J.-P. Brasselet e M.-H. Schwartz em [2] utilizando teoria de obstrução, neste trabalho os autores provaram que as classes de Schwartz de uma variedade singular coincide com as classes de MacPherson.

Usando a definição de R. D. MacPherson, a obstrução de Euler não é facilmente computável, o que motivou a obtenção de fórmulas que facilitassem o seu cálculo. Em [4], os autores apresentaram uma fórmula de natureza topológica para a obstrução de Euler, mais precisamente essa fórmula diz que a obstrução de Euler de $X$ em 0 satisfaz:

$$
E u_{X}(0)=\sum_{i} \chi\left(V_{i} \cap \mathbb{B}_{\varepsilon} \cap \ell^{-1}\left(t_{0}\right)\right) \cdot E u_{X}\left(V_{i}\right)
$$

onde $X \subset U$ é um representante de $(X, 0)$ germe de espaço analítico complexo, equidimensional, com $U$ aberto de $\mathbb{C}^{n},\left\{V_{i}\right\}$ uma estratificação de Whitney de $X, \ell: \mathbb{C}^{n} \rightarrow \mathbb{C}$ uma forma linear genérica, $\mathbb{B}_{\varepsilon}$ é uma pequena bola fechada em torno da origem em $\mathbb{C}^{n}$, $t_{0} \in \mathbb{C} \backslash\{0\}$ está suficientemente próximo a origem e $E u_{X}\left(V_{i}\right)$ é a obstrução de Euler de $X$ em qualquer ponto do estrato $V_{i}$. 
Esta fórmula é na realidade equivalente a dizer que a obstrução de Euler, como uma função construtível em X, satisfaz a chamada condição local de Euler com respeito a uma forma linear genérica.

Uma sequência natural deste resultado é o trabalho [1], de J.-P. Brasselet, D. Massey, A. J. Parameswaran e J. Seade, onde um dos objetivos é investigar o que impede a obstrução de Euler de satisfazer a condição local de Euler se trocarmos a forma linear genérica por uma função $f$ com singularidade isolada na origem. Este "defeito" que aparece na nova fórmula é chamado de obstrução de Euler de uma função.

Em certos aspectos, a obstrução de Euler de uma função é uma generalização do importante invariante da Teoria de Singularidades, o número de Milnor.

No capítulo 1, apresentaremos algumas definições básicas que serão utilizadas ao longo do texto. Procuramos nos limitar ao que realmente é necessário para a compreensão dos capítulos seguintes.

Nosso objetivo no capítulo 2 é expor os estudos referentes à obstrução de Euler de uma função. A seção 2.2 traz as definições de modificação de Nash $\tilde{X}$ de $X$ e de fibrado de Nash $\tilde{T}$ sobre a modificação de Nash $\tilde{X}$, que serão essenciais nas definições de obstrução de Euler e obstrução de Euler de uma função. Na seção 2.3, definiremos a obstrução de Euler e na seção 2.4 exibiremos uma demonstração de uma fórmula topológica para essa.

$\mathrm{Na}$ última seção deste capítulo, estudaremos a obstrução de Euler de uma função, denotada por $E u_{f, X}(0)$, onde $f: X \rightarrow \mathbb{C}$ é uma função analítica com singularidade isolada na origem. $E u_{f, X}(0)$ é a obstrução para se construir uma seção de $\tilde{T}$ que estenda o levantamento do campo $\bar{\nabla}_{X} f$, que é um campo homotópico ao campo gradiente conjugado de $f$ em $X$.

O capítulo 3 é totalmente dedicado à demonstração do teorema principal de [1], resultado que compara a obstrução de Euler e a obstrução de Euler de uma função pela fórmula

$$
E u_{V}(0)=\left(\sum \chi\left(V_{\alpha} \cap \mathbb{B}_{\varepsilon} \cap f^{-1}\left(t_{0}\right)\right) \cdot E u_{V}\left(V_{\alpha}\right)\right)+E u_{f, V}(0)
$$

A demonstração deste resultado é bastante técnica e por isso este capítulo conta com a demonstração de vários lemas. 


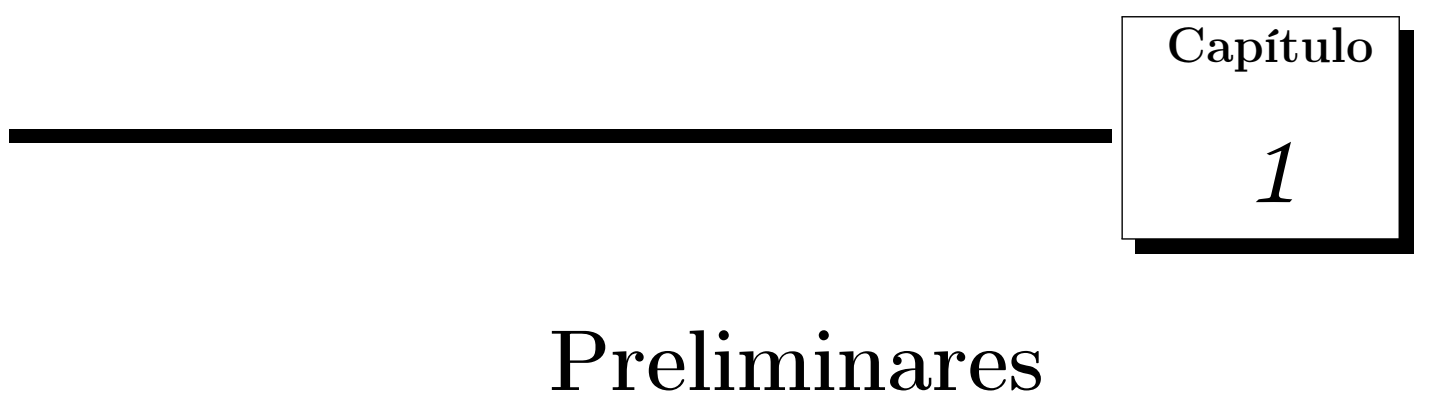

Temos como objetivo para este capítulo apresentar uma breve introdução dos conceitos que utilizaremos neste trabalho, buscando proporcionar os principais resultados necessários para a compreensão do tema que abordaremos.

\subsection{Variedades diferenciáveis}

Nesta seção, apresentaremos a definição de variedades diferenciáveis, analisaremos algumas de suas propriedades e veremos alguns conceitos importantes relacionados a variedades, que serão fundamentais para o desenvolvimento de nosso trabalho.

Definição 1.1.1. Seja $M$ um espaço topológico. Um sistema de coordenadas locais ou uma carta em $M$ é um homeomorfismo $\varphi: U \rightarrow \varphi(U)$ de um subconjunto aberto $U \subset M$ sobre um aberto $\varphi(U) \subset \mathbb{R}^{m}$.

Definição 1.1.2. Um atlas $\mathcal{U} m$-dimensional de um espaço topológico $M$ é uma família de sistemas de coordenadas locais $\varphi: U \rightarrow \varphi(U) \subset \mathbb{R}^{m}$, tal que os domínios dos sistemas de coordenadas cubram $M$. 
Definição 1.1.3. Dados os sistemas de coordenadas locais $\varphi: U \rightarrow \mathbb{R}^{m}$ e $\psi: V \rightarrow \mathbb{R}^{m}$ no espaço topológico $M$, tais que $U \cap V \neq \emptyset$, então o homeomorfismo

$$
\phi_{\varphi \psi}=\psi \circ \varphi^{-1}: \varphi(U \cap V) \rightarrow \psi(U \cap V)
$$

é chamado mudança de coordenadas.

Observação 1.1.4. Seja a mudança de coordenadas $\phi_{\varphi \psi}=\psi \circ \varphi^{-1}$, então $\left(\psi \circ \varphi^{-1}\right)^{-1}=$ $\varphi \circ \psi^{-1}=\phi_{\psi \varphi}$ é também uma mudança de coordenadas.

Definição 1.1.5. Um atlas $\mathcal{U}$ em um espaço topológico $M$ é diferenciável de classe $C^{k}$, se todas as mudanças de coordenadas $\phi_{\varphi \psi}$ forem aplicações diferenciáveis de classe $C^{k}$.

Observação 1.1.6. Da Observação 1.1.4 e da Definição 1.1.5 segue que em um atlas diferenciável $\mathcal{U}$ todas as mudanças de coordenadas $\phi_{\varphi \psi}$ são difeomorfismos.

Definição 1.1.7. Uma variedade diferenciável m-dimensional de classe $C^{k}$ é um par $(M, \mathcal{U})$, em que $M$ é um espaço topológico Hausdorff com base enumerável e $\mathcal{U}$ é um atlas $m$-dimensional de classe $C^{k}$.

Como vimos na Observação 1.1.6 em um atlas diferenciável $\mathcal{U}$ todas as mudanças de coordenadas $\phi_{\varphi \psi}$ são difeomorfismos. É exatamente esta propriedade que nos permite transportar todas as noções do Cálculo Diferencial do $\mathbb{R}^{m}$ para as variedades diferenciáveis.

Seja $M$ uma variedade de classe $C^{k}$ e seja $p$ um ponto de $M$. Indicamos por $C_{p}$ o conjunto de todos os caminhos $\lambda: I \rightarrow M$, definidos em um intervalo aberto $I$, contendo 0 , tais que $\lambda(0)=p$ e $\lambda$ é diferenciável em 0 (ver Exemplo 3, p. 132, [15]). Diremos que dois caminhos $\lambda, \mu \in C_{p}$ são equivalentes, e escreveremos $\lambda \sim \mu$, quando existir um sistema de coordenadas locais $\varphi: U \rightarrow \mathbb{R}^{m}$ em $M$, com $p \in U$, tal que $(\varphi \circ \lambda)^{\prime}(0)=(\varphi \circ \mu)^{\prime}(0)$. Como as mudanças de coordenadas são difeomorfismos, a igualdade $(\varphi \circ \lambda)^{\prime}(0)=(\varphi \circ \mu)^{\prime}(0)$ será verdadeira para todo sistema de coordenadas $\varphi: U \rightarrow \mathbb{R}^{m}$ em $M, p \in U$. Resulta daí que a relação acima é uma relação de equivalência.

O vetor velocidade $\lambda^{\prime}$ de um caminho $\lambda \in C_{p}$ é, por definição, a classe de equivalência de $\lambda$, ou seja, $\lambda^{\prime}=\left\{\mu \in C_{p}: \mu \sim \lambda\right\}$. 
Definição 1.1.8. Definimos o espaço tangente à variedade $M$ no ponto $p$ como sendo o conjunto quociente $C_{p} / \sim$. Denotaremos o espaço tangente à variedade $M$ no ponto $p$ por $T_{p} M$.

É possível mostrar que o espaço tangente à variedade diferenciável $M$ em um ponto $p \in M$ é um espaço vetorial de mesma dimensão que $M$.

Dada uma variedade diferenciável $m$-dimensional $M$, podemos obter uma nova variedade $2 m$-dimensional a partir de uma "união" dos espaços tangentes $T_{p} M$, em todos os pontos $p \in M$.

Definição 1.1.9. Seja $M$ uma variedade diferenciável de dimensão $m$. O conjunto

$$
T M=\bigcup_{p \in M}\{p\} \times T_{p} M
$$

de todos os espaços tangente $T_{p} M$, com $p \in M$, "colados" de uma maneira natural é chamado de fibrado tangente de $M$.

Proposição 1.1.10 ([14], p. 98). Dada uma variedade diferenciável m-dimensional $M$, o fibrado tangente TM tem naturalmente a estrutura de uma variedade $2 m$-dimensional.

Informalmente, o fibrado tangente de uma variedade é obtido por considerar-se todos os espaços tangentes, e reuní-los em um conjunto diferenciável e sem sobreposição, como ilustra a Figura 1.1 onde a variedade em questão é o círculo unitário em $\mathbb{R}^{2}$.
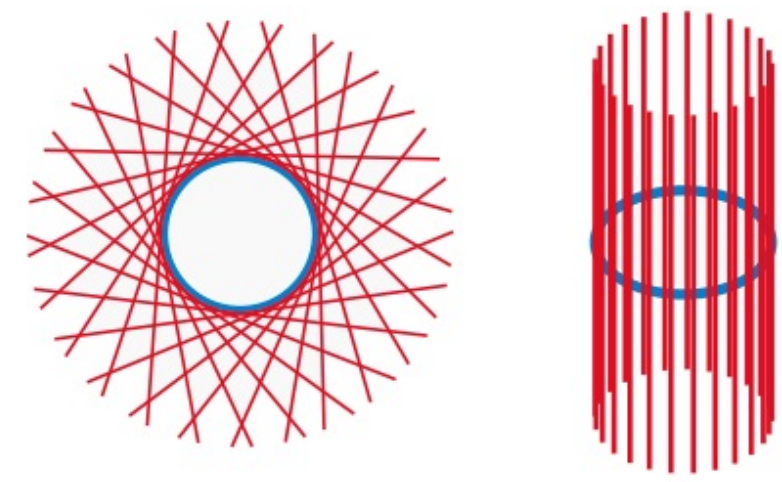

Figura 1.1: Representação do fibrado tangente de $\mathbb{S}^{1}$

Agora, que já vimos a definição de espaço vetorial tangente, podemos definir o conceito de diferenciabilidade de uma aplicação $f: M \rightarrow N$ entre variedades diferenciáveis, 
utilizando nosso conhecimento prévio a cerca das aplicações diferenciáveis definidas em um subconjunto aberto de $\mathbb{R}^{m}$ com valores em $\mathbb{R}^{n}$.

Definição 1.1.11. Sejam $M, N$ variedades diferenciáveis e uma aplicação $f: M \rightarrow N$, dizemos que $f$ é diferenciável em $p \in M$ se existem cartas $\varphi: U_{1} \rightarrow V_{1}$ para $M$ com $p \in U_{1}$ e $\psi: U_{2} \rightarrow V_{2}$ para $N$ com $f\left(U_{1}\right) \subset U_{2}$ tal que $\psi \circ f \circ \varphi^{-1}$ é diferenciável em $\varphi(p)$. Diremos simplesmente que $f$ é diferenciável se ela o for em todos os pontos de $M$. E chamaremos $f$ uma aplicação de classe $C^{k}$ quando $\psi \circ f \circ \varphi^{-1}$ for de classe $C^{k}$.

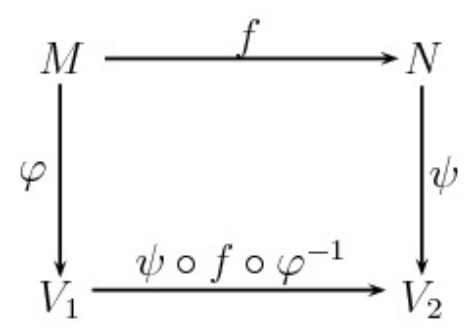

Podemos provar que a definição acima não depende das escolhas das parametrizações $\varphi$ e $\psi$, sendo então coerente.

Teorema 1.1.12 ([14], Lema 2). Seja M uma variedade diferencíavel $n$-dimensional conexa. Dados dois pontos quaisquer $p, q \in M$, existe uma carta $\varphi: V \rightarrow \mathbb{R}^{n}$ de $M$, com $p, q \in V$, tal que, $\varphi(V)=\mathbb{R}^{n}$.

Nem sempre é fácil verificar se um espaço topológico $M$ é uma variedade diferenciável, porém, existe um importante resultado relacionado ao conceito de valor regular que nos ajuda a decidir se $M$ é uma variedade diferenciável.

Definição 1.1.13. Sejam $M$ uma variedade diferenciável $m$-dimensional, $N$ uma variedade diferenciável n-dimensional e $f: M \rightarrow N$ uma aplicação diferenciável. Dizemos que:

(1) $f$ é uma imersão em $p$ se a aplicação derivada $d f_{p}$ é injetiva (portanto $n \geq m$ ) em $p \in M$. Isto é, o posto da matriz jacobiana $[J f(p)]_{n \times m}$ é igual a $m$;

(2) f é uma submersão em p se a aplicação derivada $d f_{p}$ é sobrejetora (portanto $m \geq n$ ) em $p \in M$. Isto é, o posto da matriz jacobiana $[J f(p)]_{n \times m}$ é igual a $n$. 
Se em $p \in M$ a aplicação $f: M \rightarrow N$ for uma submersão, $p$ é dito ponto regular de $f$. Um ponto $q \in N$ é chamado valor regular de $f$ se sua imagem inversa $f^{-1}(q)$ só contém pontos regulares.

Se em $p \in M$ a aplicação $f: M \rightarrow N$ não é submersão e nem imersão, então $p$ é dito ponto singular de $f$ e $f(p)$ é dito um valor singular de $f$.

Teorema 1.1.14 ([15], Proposição 1). Sejam N uma variedade diferenciável n-dimensional, $P$ uma variedade diferenciável p-dimensional, $f: N \rightarrow P$ uma aplicação diferenciável $e$ $p \in P$ um valor regular de $f$. Então, $f^{-1}(p)=M$ é uma subvariedade $(n-p)$-dimensional de $N$ e dado $q \in M$, temos $T_{q} M=\operatorname{Ker}\left(d f_{q}\right)$, onde $d f_{q}$ representa a aplicação derivada de $f$ no ponto $q$.

Dada uma aplicação $f: M \rightarrow N$ de classe $C^{1}$, existe um resultado importantíssimo acerca dos valores regulares da aplicação $f$, este resultado é conhecido como Teorema de Sard.

Teorema 1.1.15 (Teorema de Sard). Seja $f: M \rightarrow N$ uma aplicação de classe $C^{1}$, o subconjunto formado pelos valores regulares de $f$ é denso em $N$.

Sejam $M, N$ variedades diferenciáveis, $f: M \rightarrow N$ uma aplicação diferenciável e um ponto $p \in N$ vimos, no Teorema 1.1.14, que uma condição suficiente para que $f^{-1}(p)$ seja uma subvariedade de $M$ é que $p$ seja um valor regular de $f$. Deste fato é natural que façamos a seguinte pergunta: dada $S \subset N$ uma subvariedade de $N$, em que condições a imagem inversa $f^{-1}(S)$ é uma subvariedade de $M$ ? Uma resposta a esta questão é dada pela noção de transversalidade. Trata-se de uma generalização natural do conceito de valor regular.

Definição 1.1.16. Sejam $M$ uma variedade diferenciável $m$-dimensional, $N$ uma variedade diferenciável n-dimensional, $f: M \rightarrow N$ uma aplicação diferenciável e $P \subset N$ uma subvariedade $p$-dimensional de $N$, dizemos que $f$ é transversal à $P$ no ponto $q \in f^{-1}(P)$ quando

$$
d f_{q}\left(T_{q} M\right)+T_{f(q)} P=T_{f(q)} N
$$


ou seja, quando a imagem de $d f_{q}$ junto com o espaço tangente à $P$ em $f(q)$ geram todo $T_{f(q)} N$. Dizemos ainda que $f$ é transversal à $P$ se, $f$ é transversal à $P$ em $q$, para todo ponto $q \in f^{-1}(P)$.

Teorema 1.1.17 ([15], Proposição 4). Sejam M uma variedade diferenciável m-dimensional, $N$ uma variedade diferenciável $n$-dimensional, $f: M \rightarrow N$ uma aplicação diferenciável $e$ $P \subset N$ uma subvariedade p-dimensional de $N$. Se a aplicação $f$ é transversal à subvariedade $P$ então, ou $f^{-1}(P)=\varnothing$ ou $f^{-1}(P)$ é uma subvariedade $(m-n+p)$-dimensional de $M$.

Corolário 1.1.18 ([15], Corolário 1). Seja $f: M \rightarrow N$ uma submersão, então para toda variedade $P \subset N$, ou $f^{-1}(P)=\varnothing$ ou $f^{-1}(P)$ é uma subvariedade de $M$.

O conceito de transversalidade é de grande importância, tendo várias aplicações, como, por exemplo, o teorema que apresentaremos a seguir.

Teorema 1.1.19. Sejam $M$ uma variedade diferenciável $m$-dimensional e $f: M \rightarrow \mathbb{R}^{m}$ uma aplicação diferenciável. Consideremos um ponto $a \in \mathbb{R}^{m}$, um vetor $\vec{v} \neq \overrightarrow{0}$ em $\mathbb{R}^{m}$, e a reta $\Delta_{a}=\left\{a+t v: t \in \mathbb{R}^{m}\right\}$, que é uma subvariedade de $\mathbb{R}^{m}$. Definamos a aplicação $\tilde{f}: M \times \mathbb{R} \rightarrow \mathbb{R}^{m}$, dada por

$$
\tilde{f}(p, t)=f(p)+t v
$$

Nessas condições, fé transversal à $\Delta_{a}$ se, e somente se, $a \in \mathbb{R}^{m}$ é valor regular de $\tilde{f}$.

Demonstração. Seja $\varphi: V \rightarrow U$ uma carta de $M$, se $p \in V$, representaremos por $[J f(p)]_{m \times m}$ a matriz jacobiana de $f$, calculada no ponto $p$. As colunas de $[J f(p)]$ são os vetores imagens, pela aplicação linear $d f_{p}$, dos vetores da base de $T_{p} M$ associada a $\operatorname{carta} \varphi$.

Acrescentemos à direita da matriz $[J f(p)]$ a coluna formada pelas componentes do vetor $\vec{v}$ de $\mathbb{R}^{m}$, e indiquemos a matriz assim obtida com o símbolo $[J f(p), \vec{v}]_{m \times m+1}$. Observemos que $[J f(p), \vec{v}]$ é exatamente a matriz jacobiana da aplicação $\tilde{f}$, calculada no ponto $(p, t) \in M \times \mathbb{R}$. Assim, se $f$ é transversal à $\Delta_{a}$ para todo $p \in M$, tal que $f(p) \in \Delta_{a}$, então

$$
d f_{p}\left(T_{p} M\right)+T_{f(p)} \Delta_{a}=\mathbb{R}^{m}
$$


e notemos que $T_{f(p)} \Delta_{a}=\{t v: t \in \mathbb{R}\}$

Mas, dado um ponto $(p, h) \in M \times \mathbb{R}$, temos

$$
d \tilde{f}_{(p, h)}\left(T_{p} M \times \mathbb{R}\right)=d f_{p}\left(T_{p} M\right)+t v, \quad \text { onde } t \in \mathbb{R}
$$

Da condição de transversalidade, temos que $d \tilde{f}_{(p, h)}\left(T_{p} M \times \mathbb{R}\right)=d f_{p}\left(T_{p} M\right)+t v=\mathbb{R}^{m}$, onde $t \in \mathbb{R}$, daí decorre que a matriz $[J f(p), \vec{v}]$ tem posto igual $m$. Por fim notemos que, se $p \in M$ é tal que $\tilde{f}(p, t)=a$, para algum $t \in \mathbb{R}$, então $\tilde{f}(p, t)=f(p)+t \vec{v}=a$, o que implica que $f(p) \in \Delta_{a}$. Logo, o ponto $a \in \mathbb{R}^{m}$ é um valor regular de $\tilde{f}$.

Por outro lado, suponhamos que o ponto $a \in \mathbb{R}^{m}$ é valor regular de $\tilde{f}$, então para todo ponto $(p, t) \in \tilde{f}^{-1}(a)$, temos $d \tilde{f}_{(p, t)}\left(T_{p} M \times \mathbb{R}\right)=d f_{p}\left(T_{p} M\right)+t v=\mathbb{R}^{m}$. Mas, se o ponto $(p, t)$ pertence a $\tilde{f}^{-1}(a)$ significa que $f(p)=a-t v$, ou seja, que $f(p) \in \Delta_{a}$. Portanto, $f$ é transversal à $\Delta_{a}$.

Apresentaremos a seguir a definição de orientação sobre espaços vetorias. Utilizaremos esta definição e o conceito de espaço tangente para definirmos orientação sobre variedades.

Suponha que $V$ é um espaço vetorial real de dimensão finita e $\beta=\left\{v_{1}, \ldots, v_{n}\right\}$ é uma base ordenada de $V$. Se $\beta^{\prime}=\left\{v_{1}^{\prime}, \ldots, v_{n}^{\prime}\right\}$ é outra base ordenada de $V$, então existe um único isomorfismo $I: V \rightarrow V$, tal que $I(\beta)=\beta^{\prime}$, a saber

$$
I\left(\alpha_{1} v_{1}+\ldots+\alpha_{n} v_{n}\right)=\alpha_{1} v_{1}^{\prime}+\ldots+\alpha_{n} v_{n}^{\prime}
$$

Dizemos que $\beta$ e $\beta^{\prime}$ tem orientação equivalente se o determinante da transformação linear $I$ é positivo. Isto é, se escrevemos

$$
\begin{gathered}
I\left(v_{1}\right)=v_{1}^{\prime}=a_{11} v_{1}+\ldots+a_{n 1} v_{n} \\
\vdots \\
I\left(v_{n}\right)=v_{n}^{\prime}=a_{1 n} v_{1}+\ldots+a_{n n} v_{n} .
\end{gathered}
$$


então, $\beta$ e $\beta^{\prime}$ têm orientação equivalente se $\operatorname{det}[I]_{\beta}>0$, onde

$$
[I]_{\beta}=\left[\begin{array}{ccc}
a_{11} & \cdots & a_{1 n} \\
\vdots & \ddots & \vdots \\
a_{n 1} & \cdots & a_{n n}
\end{array}\right]
$$

Observemos que como $I$ é um isomorfismo $\operatorname{det}[I]_{\beta} \neq 0$. Note também, que $[I]_{\beta}=M_{\beta}^{\beta^{\prime}}$, ou seja, a matriz da transformação $I$ com relação a base $\beta$ é igual a matriz de mudança de base $\beta$ para $\beta^{\prime}$. Então, utilizando a regra do produto para determinantes e a igualdade

$$
M_{\beta}^{\beta^{\prime \prime}}=M_{\beta^{\prime}}^{\beta^{\prime \prime}} M_{\beta}^{\beta^{\prime}}
$$

temos que dadas bases $\beta, \beta^{\prime}$ e $\beta^{\prime \prime}$ de $V$ e $I_{1}, I_{2}$ os isomorfismos de $V$ em $V$, tais que $I_{1}(\beta)=\beta^{\prime}$ e $I_{2}\left(\beta^{\prime}\right)=\beta^{\prime \prime}$, temos

$$
\left[I_{2} \circ I_{1}\right]=M_{\beta}^{\beta^{\prime \prime}}=M_{\beta^{\prime}}^{\beta^{\prime \prime}} M_{\beta}^{\beta^{\prime}}
$$

$\log \mathrm{O} \operatorname{det}\left[I_{2} \circ I_{1}\right]_{\beta}=\operatorname{det}\left[I_{2}\right]_{\beta^{\prime}} \operatorname{det}\left[I_{1}\right]_{\beta}$.

Assim, este processo define uma relação de equivalência, particionando o conjunto de todas as bases ordenadas de $V$ em duas classes.

Definição 1.1.20. Uma orientação de $V$ é uma decisão arbitrária para fixar um sinal positivo para elementos de uma classe de equivalência e um sinal negativo para os outros. Um espaço vetorial orientado $V$ é um par formado pelo espaço vetorial $V$ e uma orientação de $V$. As bases de $V$ pertencentes a esta orientação serão chamadas de positivas e as outras de negativas.

Exemplo 1.1.21. Por exemplo, o espaço euclidiano $\mathbb{R}^{n}$ será sempre orientado positivamente pela base canônica.

Observação 1.1.22. Dado $V$ um espaço vetorial real de dimensão finita, quando falamos de uma orientação de $V$ é indispensável considerarmos as suas bases ordenadas. Por exemplo, se considerarmos $\beta=\left\{e_{1}, e_{2}\right\}$ como sendo uma base positiva de $\mathbb{R}^{2}$, onde 
$e_{1}=(1,0)$ e $e_{2}=(0,1)$, então a base $\beta^{\prime}=\left\{e_{1}^{\prime}, e_{2}^{\prime}\right\}$, com $e_{1}^{\prime}=(0,1)$ e $e_{2}^{\prime}=(-1,0)$, é equivalente a $\beta$, porém a base $\alpha=\left\{f_{1}, f_{2}\right\}$, com $f_{1}=(-1,0)$ e $f_{2}=(0,1)$, não é equivalente a base $\beta$.

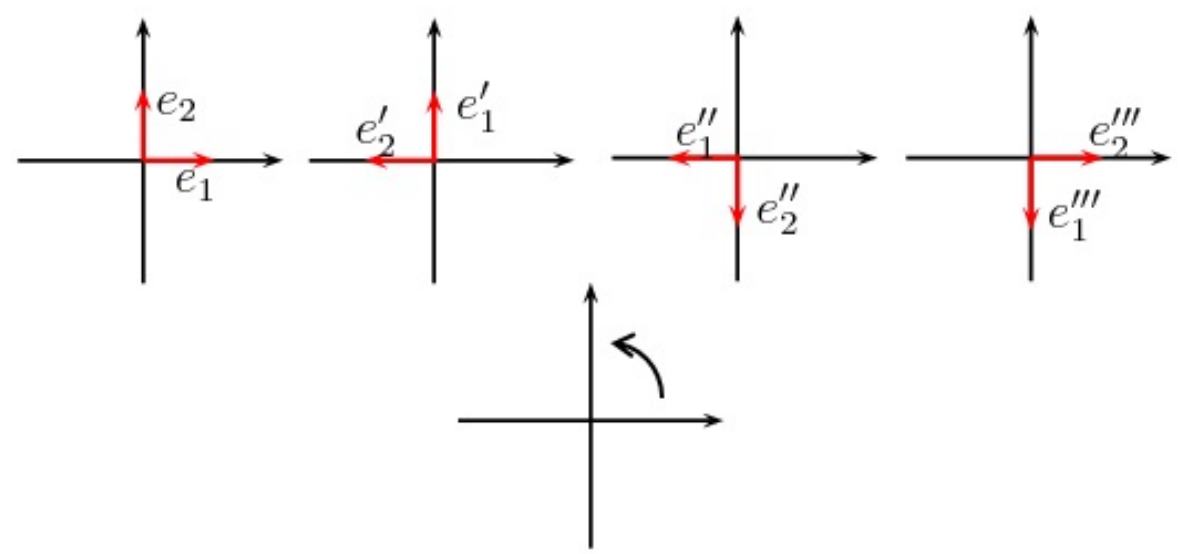

Figura 1.2: Exemplo de orientação em $\mathbb{R}^{2}$

Dados $V$ e $W$ dois espaços vetoriais de mesma dimensão $n$, se $I: V \rightarrow W$ é um isomorfismo de $V$ em $W$ sabemos que $I$ leva uma base de $V$ em uma base de $W$. Assim, temos a seguinte definição.

Definição 1.1.23. Sejam $V$ e $W$ dois espaços vetoriais $n$-dimensionais e orientados, se $I: V \rightarrow W$ é um isomorfismo de $V$ em $W$, dizemos que $I$ preserva orientação se I leva base positiva de $V$ em uma base positiva de $W$, ou equivalentemente se I leva base negativa de $V$ em uma base negativa de $W$. Caso contrário, diremos que I inverte orientação. Quando um isomorfismo entre espaços vetoriais orientados preserva (inverte) orientação, dizemos também que esse isomorfismo é positivo (negativo).

Agora, voltemos para variedades.

Definição 1.1.24. Seja $M$ uma variedade diferenciável $m$-dimensional, dizemos que $M$ é orientável se existe uma escolha contínua de orientações para todos os espaços tangentes $T_{p}$ M. A condição de continuidade pode ser interpretada no seguinte sentido: próximo de cada ponto $p \in M$ deve existir uma carta $\varphi: U \rightarrow V$, com $p \in U$, tal que $d \varphi_{u}: T_{u} M \rightarrow \mathbb{R}^{m}$ preserva orientação em cada ponto $u \in U$. Uma variedade orientada é um par $(M, \mathcal{O})$ onde $M$ é uma variedade diferenciável e $\mathcal{O}$ é uma orientação em $M$. 
Exemplo 1.1.25. A circunferência unitária $\mathbb{S}^{n} \subset \mathbb{R}^{n+1}$ é orientável.

Exemplo 1.1.26. Dada uma variedade diferenciável $M$, o fibrado tangente $T M$ de $M$ é uma variedade diferenciável orientável, quer $M$ o seja, quer não.

Observação 1.1.27. Dada uma variedade diferenciável $M m$-dimensional, já vimos que para cada ponto $p \in M$ obtemos o espaço vetorial $T_{p} M$. Dada $\varphi: V \rightarrow U$ uma carta de $M \operatorname{com} p \in V$, denotaremos por $\left\{\frac{\partial}{\partial x_{1}}(p), \ldots, \frac{\partial}{\partial x_{m}}(p)\right\}$ a base $\left\{d \varphi_{\varphi(p)}^{-1}\left(e_{1}\right), \ldots, d \varphi_{\varphi(p)}^{-1}\left(e_{m}\right)\right\}$ de $T_{p} M$, onde $\left\{e_{1}, \ldots, e_{m}\right\}$ denota a base canônica de $\mathbb{R}^{m}$ e $\varphi(p)=\left(x_{1}, \ldots, x_{m}\right) \in \mathbb{R}^{m}$. Além disso, consideraremos o espaço vetorial $T_{p} M$ positivamente orientado pela base $\left\{\frac{\partial}{\partial x_{1}}(p), \ldots, \frac{\partial}{\partial x_{m}}(p)\right\}$, salvo o caso em que digamos o contrário.

Definição 1.1.28. Sejam $M, N$ variedades diferenciáveis $n$-dimensionais, orientadas e $f: M \rightarrow N$ uma aplicação diferenciável. Dados um valor regular $p \in N$ de $f$ e os pontos regulares $q \in f^{-1}(p)$ diremos que o ponto $q$ é positivo $(q>0)$ se o isomorfismo $d f_{q}: T_{q} M \rightarrow T_{p} N$ preserva a orientação e diremos que o ponto q é negativo $(q<0)$ se o isomorfismo $d f_{q}: T_{q} M \rightarrow T_{p} N$ inverte a orientação.

Teorema 1.1.29. Sejam $M$ e $N$ variedades diferenciáveis m-dimensionais orientadas, com $M$ conexa. Se $f: M \rightarrow N$ é um difeomorfismo local, então $d f_{x}: T_{x} M \rightarrow T_{f(x)} N$ preserva ou inverte orientação, para todo $x \in M$.

Apresentaremos a seguir, o conceito de variedades diferenciáveis com bordo. Uma referência deste assunto é [5].

Definição 1.1.30. Uma variedade diferenciável m-dimensional com bordo é um espaço topológico $M$ com uma coleção de aplicações $\varphi: V \rightarrow U$, onde $V$ é um aberto de $M$ e $U$ é um aberto do subconjunto $\mathbb{H}^{m}=\left\{\left(x_{1}, \ldots, x_{m}\right): x_{m} \geq 0\right\}$, satisfazendo as condições da definição de variedades diferenciáveis (ver Definição 1.1.7).

Dizemos que $p \in M$ é um ponto do bordo de $M$, se existe $\varphi: V \rightarrow U$ tal que $\varphi(p)=q$ onde $q \in \partial \mathbb{H}^{m}=\left\{\left(x_{1}, \ldots, x_{m}\right): x_{m}=0\right\}$.

$O$ bordo de uma variedade diferenciável $m$-dimensional com bordo $M$ será denotado por $\partial M$. 
Definição 1.1.31. Uma variedade diferenciável fechada é uma variedade compacta e sem bordo.

Podemos definir também o conceito de variedades complexas. Uma referência deste assunto é [20].

Definição 1.1.32. Dizemos que uma variedade diferenciável $M$ de dimensão $2 m$ é uma variedade complexa de dimensão $m$, se $M$ tem um atlas formado apenas por cartas em $\mathbb{C}^{m}$, tais que a mudança de coordenadas entre estas cartas seja holomorfa.

Dado que aplicações holomorfas são muito mais especiais que as aplicações diferenciáveis, temos que a teoria de variedades diferenciáveis e variedades complexas têm aspectos muito diferentes. O teorema a seguir, cuja demonstração pode ser vista em [20], serve de ilustração para este fato.

Teorema 1.1.33 ([20], Lema 14.1). Toda variedade complexa M é orientável.

\section{Índice de Poincaré-Hopf}

Nesta seção, consideraremos $M, N$ variedades diferenciáveis n-dimensionais, orientadas e $f: M \rightarrow N$ uma aplicação diferenciável. Se $M$ é compacta e $N$ é conexa, então definiremos o grau de $f$, para isto considere a seguinte definição.

Definição 1.1.34. Sejam $M$ variedade fechada e $N$ uma variedade conexa. Seja $x \in M$ um ponto regular de $f$ tal que $d f_{x}: T_{x} M \rightarrow T_{f(x)} N$ é um isomorfismo entre espaços vetoriais orientados. Defina o sinal de $d f_{x}$, denotado por sign $d f_{x}$, como sendo 1 se $d f_{x}$ preserva orientação ou -1 se $d f_{x}$ inverte orientação. Para todo valor regular $y \in N$ definimos

$$
\operatorname{grau}(f, y)=\sum_{x \in f^{-1}(y)} \operatorname{sign} d f_{x}
$$

Teorema 1.1.35 ([19], Teorema A). O inteiro grau $(f, y)$ não depende da escolha do valor regular $y$. 
Definição 1.1.36. Definimos o grau de $f$, denotado por grau $(f)$, como sendo $\operatorname{grau}(f, y)$, para algum valor regular $y$.

Definição 1.1.37. Considere um conjunto aberto $U \subset \mathbb{R}^{n}$ e um campo vetorial contínuo

$$
v: U \rightarrow \mathbb{R}^{n}
$$

com uma singularidade isolada em $a \in U$. A aplicação

$$
\phi(x)=\frac{v(x)}{\|v(x)\|}
$$

aplica uma pequena esfera de centro em a na esfera unitária.

O grau da aplicação $\phi$ definida acima é chamado de indice de Poincaré-Hopf de v na singularidade a, que denotaremos por $\operatorname{Ind}_{P H}(v, a)$.

Definição 1.1.38. Sejam $M$ variedade fechada e $N$ uma variedade conexa. Os campos de vetores $v$ em $M$ e $v^{\prime}$ em $N$ correspondem sobre $f$ se a derivada df leva $v(x)$ sobre $v^{\prime}(f(x))$ para cada $x \in M$.

Se $f$ é um difeomorfismo, então $v^{\prime} e ́$ unicamente determinado por $v$, usaremos a notação

$$
v^{\prime}=d f \circ v \circ f^{-1}
$$

Lema 1.1.39 ([19], Lema 1). Se o campo de vetores $v$ em $U \subset \mathbb{R}^{n}$ corresponde a $v^{\prime}=d f \circ v \circ f^{-1}$ em $U^{\prime} \subset \mathbb{R}^{n}$ sobre um difeomorfismo $f: U \rightarrow U^{\prime}$. Então, o indice de Poincaré-Hopf de v em uma singularidade isolada a é igual ao indice de Poincaré-Hopf de $v^{\prime}$ em $f(a)$.

Assumindo o Lema 1.1.39, podemos definir o conceito de índice de Poincaré-Hopf para um campo de vetores $w$ em uma variedade arbitrária $M$.

Definição 1.1.40. Se $g: U \rightarrow M$ é uma parametrização de uma vizinhança de a em $M$, então o índice de Poincaré-Hopf de $w$ em a, denotado por $\operatorname{Ind}_{P H}(w, a)$, é o índice do campo de vetores correspondente $d g^{-1} \circ w \circ g$ em $U$ na singularidade $g^{-1}(a)$. 
Teorema 1.1.41 (Teorema de Poincaré-Hopf). Seja $M$ uma variedade fechada e v um campo de vetores contínuo em $M$ com singularidades isoladas. Então, a soma dos indices de Poincaré-Hopf das singularidades de v é igual a característica de Euler de $M$, isto é,

$$
\chi(M)=\sum_{a} \operatorname{Ind}_{P H}(v, a)
$$

Podemos citar como uma referência para a demonstração deste teorema [19]. Temos o Teorema de Poincaré-Hopf para o caso em que $M$ é uma variedade compacta orientada com bordo, a demonstração deste resultado pode ser vista em [3].

Teorema 1.1.42. Sejam $M$ uma variedade diferenciável, compacta, orientada e com bordo $\partial M$, e $v$ um campo de vetores sem singularidades em uma vizinhança $U$ de $\partial M$. Então:

i) O campo de vetores $v$ pode ser estendido para o interior de $M$ com uma quantidade finita de singularidades;

ii) A soma dos índices de Poincaré-Hopf das singularidade de $v$ em $M$, independe da maneira como estendemos o campo $v$ para o interior de $M$;

iii) Seja $\tilde{v}$ uma extensão de $v$ em $M$, se $\tilde{v}$ é transversal ao bordo de $M$ e aponta para "fora" de $M$, então temos que

$$
\chi(M)=\sum_{a} \operatorname{Ind}_{P H}(\tilde{v}, a)
$$

Se $\tilde{v}$ é transversal a $\partial M$ e aponta para "dentro" de $M$, então

$$
\chi(M)-\chi(\partial M)=\sum_{a} \operatorname{Ind}_{P H}(\tilde{v}, a) .
$$




\subsection{Homotopia}

Nesta seção introduziremos noções básicas sobre homotopia, procurando ilustrá-las com alguns exemplos.

Definição 1.2.1. Sejam $X$ e $Y$ espaços topológicos, dizemos que duas aplicações contínuas $f, g: X \rightarrow Y$ são homotópicas quando existe uma aplicação contínua

$$
H: X \times I \rightarrow Y, \quad \text { com } I=[0,1]
$$

tal que $H(x, 0)=f(x)$ e $H(x, 1)=g(x)$ para todo $x \in X$. Chamaremos a aplicação $H$ de homotopia entre $f$ e $g$ e escreveremos $f \simeq g$.

Exemplo 1.2.2. Sejam $E$ um espaço vetorial normado, $Y \subset E$ um espaço topológico com a topologia induzida de $E$ e $X$ um espaço topológico qualquer. Dadas aplicações contínuas $f, g: X \rightarrow Y$, suponhamos que, para todo $x \in X$, o segmento de reta $[f(x), g(x)]$ esteja contido em $Y$. Então, $f \simeq g$.

De fato, denotando o intervalo [0,1] por $I$, basta definirmos $H(x, t)=(1-t) f(x)+\operatorname{tg}(x)$ para obtermos uma homotopia $H: X \times I \rightarrow Y$ entre $f$ e $g$.

Em particular, dada uma aplicação constante

$$
\begin{gathered}
f: X \quad \longrightarrow \quad E \\
x \longmapsto c
\end{gathered}
$$

toda aplicação contínua $g: X \rightarrow E$ é homotópica à $f$.

Exemplo 1.2.3. Seja $\mathbb{S}^{n}$ a esfera unitária de $\mathbb{R}^{n+1}$, dadas duas aplicações contínuas $f, g: X \rightarrow \mathbb{S}^{n}$, se $f(x) \neq-g(x)$ para todo $x \in X$, isto é, se $f(x)$ e $g(x)$ nunca são pontos antípodas, então $f \simeq g$.

Proposição 1.2.4 ([14], Lema 1). Sejam X e Y espaços topológicos. A relação de homotopia $f \simeq g$ é uma relação de equivalência no conjunto das aplicações contínuas de $X$ e $Y$. 
Proposição 1.2.5. Sejam $X, Y$ e $Z$ espaços topológicos, consideremos aplicações $f, f^{\prime}: X \rightarrow Y$ e $g, g^{\prime}: Y \rightarrow Z$ aplicações contínuas. $S e f \simeq f^{\prime}$ e $g \simeq g^{\prime}$, então $g \circ f \simeq g^{\prime} \circ f^{\prime}$.

Demonstração. Seja $I=[0,1]$, denotemos por $H: X \times I \rightarrow Y$ uma homotopia entre $f$ e $f^{\prime}$ e por $K: Y \times I \rightarrow Z$ uma homotopia entre $g$ e $g^{\prime}$. Definindo $L: X \times I \rightarrow Z$ por $L(x, t)=K(H(x, t), t)$, temos que $L$ é uma homotopia entre $g \circ f$ e $g^{\prime} \circ f^{\prime}$.

Existe uma estreita relação entre homotopia e o problema de estender continuamente a todo o espaço uma aplicação contínua definida em um subconjunto fechado desse espaço. Um exemplo disto pode ser visto na seguinte proposição.

Proposição 1.2.6 ([14, Proposição 1). Sejam X um espaço topológico e $\overline{\mathbb{B}}_{1}(0)$ a bola fechada de centro 0 e raio 1 de $\mathbb{R}^{n+1}$. Uma aplicação contínua $f: \mathbb{S}^{n} \rightarrow X$ estende-se continuamente à $\overline{\mathbb{B}}_{1}(0)$ se, e somente se, é homotópica à uma constante.

Demonstração. Denotemos por $I$ o intervalo $[0,1]$ e consideremos a aplicação

$$
\begin{aligned}
\varphi: \mathbb{S}^{n} \times I & \longrightarrow \overline{\mathbb{B}}_{1}(0) \\
(x, t) & \longmapsto(1-t) x
\end{aligned}
$$

que é contínua, sobrejetora e igual a 0 em $\mathbb{S}^{n} \times 1$. Se $\tilde{f}: \overline{\mathbb{B}}_{1}(0) \rightarrow X$ é uma extensão contínua de $f: \mathbb{S}^{n} \rightarrow X$, então

$$
H=\tilde{f} \circ \varphi: \mathbb{S}^{n} \times I \rightarrow X
$$

é uma homotopia entre $f$ e a aplicação constante $g: \mathbb{S}^{n} \rightarrow X$, definida por $g(x)=\tilde{f}(0)$. Reciprocamente, suponhamos que $H: \mathbb{S}^{n} \times I \rightarrow X$ seja uma homotopia entre $f$ e uma aplicação constante $g: \mathbb{S}^{n} \rightarrow X$, dada por $g(x)=p$, para todo $x \in \mathbb{S}^{n}$. Seja a aplicação $\tilde{f}: \overline{\mathbb{B}}_{1}(0) \rightarrow X$ definida da seguinte maneira,

$$
\tilde{f}(x)=\left\{\begin{array}{l}
H\left(\frac{x}{|x|}, 1-|x|\right), \text { se } x \neq 0 \\
p, \text { se } x=0
\end{array}\right.
$$

Assim, temos que $\tilde{f}$ é contínua e $\left.\tilde{f}\right|_{\mathbb{S}^{n}}=f$. 
A seguir apresentamos um resultado, cuja demonstração pode ser vista em [14].

Teorema 1.2.7 ([14], Teorema 1). Sejam $M$ e $N$ variedades diferenciáveis compactas, então toda aplicação contínua $f: M \rightarrow N$ é homotópica à uma aplicação $g: M \rightarrow N$ de classe $C^{1}$.

\subsection{Homologia singular}

Nesta seção apresentaremos uma análise da teoria de homologia singular. Analisaremos os R-módulos de homologia singular com coeficientes em um anel R comutativo com identidade. Uma referência para este assunto é [21].

\section{Simplexos em $\mathbb{R}^{n}$}

Definição 1.3.1. Dados $(p+1)$ pontos em $\mathbb{R}^{n}, p \leq n$, dizemos que $\left\{X_{0}, \ldots, X_{p}\right\}$ é uma coleção geometricamente independente se os vetores $\overrightarrow{v_{1}}=X_{1}-X_{0}, \overrightarrow{v_{2}}=X_{2}-X_{0}, \ldots$, $\overrightarrow{v_{p}}=X_{p}-X_{0}$ são linearmente independentes.

Definição 1.3.2. Seja $\left\{X_{0}, \ldots, X_{p}\right\} \subset \mathbb{R}^{n}$ uma coleção geometricamente independente de $\mathbb{R}^{n}, p \leq n$. O p-simplexo $S$ gerado por $X_{0}, \ldots, X_{p}$ é a envoltória convexa:

$$
S=E C\left(\left\{X_{0}, \ldots, X_{p}\right\}\right)
$$

Os pontos $X_{i}$ são chamados vértices do p-simplexo $S$.

Teorema 1.3.3 (Teorema das coordenadas baricêntricas). Sejam $\left\{X_{0}, \ldots, X_{p}\right\}$ uma coleção de $(p+1)$ pontos geometricamente independentes em $\mathbb{R}^{n}$, com $p \leq n$. Considere o seguinte conjunto:

$$
A_{p}=\left\{t_{0} X_{0}+\ldots+t_{p} X_{p} ; \quad 0 \leq t \leq 1 \quad e \sum_{i=0}^{p} t_{i}=1\right\} \subset \mathbb{R}^{n}
$$

(i) $A_{p}=E C\left(\left\{X_{0}, \ldots, X_{p}\right\}\right)=$ p-simplexo gerado por $X_{0}, \ldots, X_{p}$; 
(ii) $S e \sum_{i=0}^{p} t_{i} X_{i}=\sum_{i=0}^{p} s_{i} X_{i}, \quad 0 \leq t_{i}, s_{i} \leq 1$ com $\sum_{i=0}^{p} t_{i}=\sum_{i=0}^{p} s_{i}=1$, então $t_{i}=s_{i}$ para todo $i=1, \ldots, p$, ou seja: todo elemento de $A_{p}$ se escreve de maneira única como combinação linear dos pontos $\left\{X_{0}, \ldots, X_{p}\right\}$.

Observação 1.3.4. Concluímos que escolhida uma ordenação $\left\{X_{0}, \ldots, X_{p}\right\}$ para $(p+1)$ pontos geometricamente independentes em $\mathbb{R}^{n}$, com $p \leq n$, cada ponto $X \in E C\left(\left\{X_{0}, \ldots, X_{p}\right\}\right)$ é univocamente determinado por uma $(p+1)$-upla $\left(t_{0}, t_{1}, \ldots, t_{p}\right)$, com $0 \leq t_{i} \leq 1$ e $\sum_{i=0}^{p} t_{i}=1$ denominada as coordenadas baricêntricas do ponto X.

Definição 1.3.5. Sejam $X_{0}=e_{1}=(1,0, \ldots, 0), X_{1}=e_{2}=(0,1,0, \ldots, 0), \ldots$, $X_{p}=e_{p+1}=(0,0, \ldots, 0,1)$. O p-simplexo $E C\left(\left\{X_{0}, \ldots, X_{p}\right\}\right)$, nesse caso, é denominado p-simplexo padrão e será denotado por $\Delta_{p} \in \mathbb{R}^{p+1}$

\section{Homologia singular de um espaço topológico $X$ com coeficientes em um anel $R$}

Fixemos $R$ um anel comutativo com a identidade $1_{R}$ e consideremos $\Delta_{p} \subset \mathbb{R}^{p+1} \mathrm{o}$ $p$-simplexo padrão munido da topologia induzida de subespaço em $\mathbb{R}^{p+1}$ (munido da topologia usual).

Definição 1.3.6. Para cada espaço topológico $X$, um p-simplexo singular em $X$ é uma função contínua $\sigma: \Delta_{p} \rightarrow X$.

Denotaremos por

$$
C_{p}(X)=\left\{\sigma: \Delta_{p} \rightarrow X ; \quad \sigma \text { é contínua }\right\}
$$

o conjunto de todos os $p$-simplexos singulares de $X$.

Definição 1.3.7. Para cada $p \geq 0$, definimos o R-módulo livre cuja base é o conjunto de todos os $p$-simplexos singulares de $X$ :

$S_{p}\left(C_{p}(X), R\right)=\left\{f: C_{p}(X) \rightarrow R ; f(\sigma) \neq 0\right.$, apenas para um número finito de elementos $\left.\sigma\right\}$. 
Um elemento típico de $S_{p}\left(C_{p}(X), R\right)$ é uma combinação linear formal $\alpha_{1} \sigma_{1}+\ldots+\alpha_{r} \sigma_{r}$, onde $\alpha_{i} \in R$ e $\sigma_{i}: \Delta_{p} \rightarrow X$ é um $p$-simplexo singular de $X$.

$$
\begin{aligned}
\sum_{i=1}^{r} \alpha_{i} \sigma_{i} \longleftrightarrow f: C_{p}(X) & \rightarrow R \\
\sigma & \mapsto f(\sigma)= \begin{cases}\alpha_{i} ; & \text { se } \sigma=\sigma_{i} i=1, \ldots, r \\
0, & \text { se } \sigma \neq \sigma_{i}\end{cases}
\end{aligned}
$$

Os elementos de $S_{p}\left(C_{p}(X) ; R\right)$ são chamados $p$-cadeias singulares de X com coeficientes em R.

A seguir, definiremos o complexo de cadeias $\left\{S_{p}(X ; R) ; \partial\right\}_{p \geq 0}$. Para isso, convencionaremos que $\left\{S_{p}(X ; R)\right\}=\{0\}$ para todo $p<0$.

\section{Os operadores face}

Definição 1.3.8. Seja $\sigma: \Delta_{p} \rightarrow X$ um p-simplexo singular de $X$. Considere a inclusão

$$
\begin{array}{ccc}
\Delta_{p-1} & \stackrel{\varepsilon_{i}}{\hookrightarrow} & \Delta_{p} \\
\left(t_{0}, \ldots, t_{i}, \ldots, t_{p-1}\right) & \hookrightarrow & \left(t_{0}, \ldots, t_{i-1}, 0, t_{i}, \ldots, t_{p-1}\right),
\end{array}
$$

que insere o zero na i-ésima posição. Para cada $i=1, \ldots, p$ definimos a i-ésima face de $\sigma$ como sendo o $(p-1)$-simplexo singular de $X$ dado pela composição

$$
\sigma \circ \varepsilon_{i}: \Delta_{p-1} \rightarrow X
$$

que é contínua e denotada por $\partial_{i} \sigma: \Delta_{p-1} \rightarrow X$.

Assim, para cada $i=1, \ldots, p$ e para cada $p$-simplexo singular $\sigma \in C_{p}(X)$, associamos a sua i-ésima face $\partial_{i} \sigma \in C_{p-1}(X)$. Isso define uma função

$$
\begin{array}{ccc}
\partial_{i}: C_{p}(X) & \longrightarrow & C_{p-1}(X) \subset S_{p-1}(X ; R) \\
\sigma & \longmapsto & \partial_{i} \sigma
\end{array}
$$


para todo $i=1, \ldots, p$, e desde que $C_{p}(X)$ é base para o R-módulo livre $S_{p}(X ; R), \partial_{i}$ se estende por linearidade a um único R-homomorfismo $\partial_{i}: S_{p}(X ; R) \rightarrow S_{p-1}(X ; R)$, chamado operador face. O R-homomorfismo $\partial: S_{p}(X ; R) \rightarrow S_{p-1}(X ; R)$ dado pela soma alternada dos operadores face

$$
\partial=\partial_{0}-\partial_{1}+\partial_{2}-\ldots+(-1)^{p} \partial_{p}=\sum_{i=0}^{p}(-1)^{i} \partial_{i}
$$

é chamado operador bordo.

Teorema 1.3.9 ([21], Teorema 29.1). O operador $\partial: S_{p}(X ; R) \rightarrow S_{p-1}(X ; R)$, definido por $\sum_{i=0}^{p}(-1)^{i} \partial_{i}$, é tal que $\partial \circ \partial=0$.

Definição 1.3.10. Fixado um anel comutativo com identidade $R$ para cada espaço topológico $X$, criamos um complexo de cadeias $S_{*}(X ; R)=\left\{S_{p}(X ; R), \partial\right\}_{p \geq 0}$, ou seja,

$$
S_{*}(X ; R): \cdots \stackrel{\partial}{\longrightarrow} S_{p}(X ; R) \stackrel{\partial}{\longrightarrow} S_{p-1}(X ; R) \stackrel{\partial}{\longrightarrow} \cdots \stackrel{\partial}{\longrightarrow} S_{1}(X ; R) \stackrel{\partial}{\longrightarrow} S_{0}(X ; R) \stackrel{\partial}{\longrightarrow} 0,
$$

usaremos as seguintes terminologias:

$Z_{p}\left(S_{*}(X ; R)\right) \doteq Z_{p}(X ; R)=\operatorname{Ker}(\partial)$ o submódulo dos p-ciclos,

$B_{p}\left(S_{*}(X ; R)\right) \doteq B_{p}(X ; R)=\operatorname{Im}(\partial)$ o submódulo dos $p$-bordos e

$H_{p}\left(S_{*}(X ; R)\right) \doteq H_{p}(X ; R)=\frac{Z_{p}(X ; R)}{B_{p}(X ; R)}$ o p-ésimo $R$-módulo de homologia de $X$ com coeficientes em $R$.

Um elemento típico de $H_{p}(X ; R)$ é da forma

$$
\mu=z_{p}+B_{p}(X ; R)
$$

onde $z_{p} \in Z_{p}(X ; R)$ é um $p$-ciclo; $\mu$ é chamada classe de homologia de $\mathrm{X}$ com coeficientes em $\mathrm{R}$, representada pelo p-ciclo $z_{p}$. 


\section{Construção do R-homomorfismo induzido em homologia}

Dada uma função contínua $f: X \rightarrow Y$, queremos definir um $R$-homomorfismo $f_{*}: H_{p}(X ; R) \rightarrow H_{p}(Y ; R)$ para cada $p \geq 0$. Para isso, precisamos definir uma aplicação de cadeias

$$
f_{\#}: S_{p}(X ; R) \rightarrow S_{p}(Y ; R)
$$

para cada $p \geq 0$, entre os complexos de cadeias $S_{p}(X ; R)$ e $S_{p}(Y ; R)$.

Observemos que, dado um $p$-simplexo singular de $X, \sigma: \Delta_{p} \rightarrow X$, a composição

$$
f \circ \sigma: \Delta_{p} \stackrel{\sigma}{\longrightarrow} X \stackrel{f}{\longrightarrow} Y
$$

é um $p$-simplexo singular de $Y$ e determina uma função

$$
\begin{aligned}
C_{p}(X) & \longrightarrow C_{p}(Y) \quad \subset S_{p}(Y ; R) \\
\sigma & \longmapsto f \circ \sigma
\end{aligned}
$$

para cada $p \geq 0$. Desde que $C_{p}(X)$ é base para o $R$-módulo livre $S_{p}(X ; R)$ e $C_{p}(Y) \subset S_{p}(Y ; R)$ tal função se estende por linearidade a um único $R$-homomorfismo $f_{\#}: S_{p}(X ; R) \rightarrow S_{p}(Y ; R)$, definido por

$$
f_{\#}\left(\alpha_{1} \sigma_{1}+\ldots+\alpha_{r} \sigma_{r}\right)=\alpha_{1}\left(f \circ \sigma_{1}\right)+\ldots+\alpha_{r}\left(f \circ \sigma_{r}\right) .
$$

Teorema 1.3.11 ([21], Teorema 29.1). O R-homomorfismo $f_{\#}: S_{p}(X ; R) \rightarrow S_{p}(Y ; R)$ é uma aplicação de cadeias.

Portanto, $f_{\#}: S_{p}(X ; R) \rightarrow S_{p}(Y ; R)$ induz um bem definido $R$-homomorfismo $f_{*}: H_{p}(X ; R) \rightarrow H_{p}(Y ; R)$, dado por

$$
f_{*}\left(z_{p}+B_{p}(X ; R)\right)=f_{\#}\left(z_{p}\right)+B_{p}(Y ; R),
$$

onde

$$
f_{\#}\left(z_{p}\right)=f_{\#}\left(\alpha_{1} \sigma_{1}+\ldots+\alpha_{r} \sigma_{r}\right)=\alpha_{1}\left(f \circ \sigma_{1}\right)+\ldots+\alpha_{r}\left(f \circ \sigma_{r}\right) .
$$




\subsection{Cohomologia}

Nesta seção apresentaremos alguns conceitos sobre cohomologia que serão necessários no decorrer deste texto. Uma referência para este assunto é [21].

\section{Axiomas de Cohomologia}

Dados um conjunto $\mathcal{A}$ de pares de espaços topológicos $(X, A)$ e um grupo abeliano $\mathrm{G}$, uma Teoria de Cohomologia em $\mathcal{A}$ com coeficientes em $\mathrm{G}$ consiste de:

(1) Uma função definida para cada inteiro $p$ e para cada par $(X, A)$ que associa a estes um grupo abeliano $H^{p}(X, A ; G)$;

(2) Uma função contínua $h:(X, A) \rightarrow(Y, B)$ a qual induz um homomorfismo

$$
h^{p}: H^{p}(Y, B ; G) \rightarrow H^{p}(X, A ; G)
$$

(3) Para cada par (X,A) tem-se um homomorfismo

$$
\delta^{p}: H^{p-1}(A ; G) \rightarrow H^{p}(X, A ; G) .
$$

Satisfazendo os seguintes axiomas:

(1) Se $i:(X, A) \rightarrow(X, A)$ é a identidade, então $i^{p}: H^{p}(X, A ; G) \rightarrow H^{p}(X, A ; G)$ também o é, para todo inteiro p;

(2) Dadas $h:(X, A) \rightarrow(Y, B)$ e $k:(Y, B) \rightarrow(Z, C)$, temos $(k \circ h)^{p}=h^{p} \circ k^{p}$, para todo inteiro p;

(3) Dada $f:(X, A) \rightarrow(Y, B)$ contínua, $\delta^{p}$ é uma aplicação natural, isto é, o diagrama a seguir comuta: 


$$
\begin{array}{ccc}
H^{p}(X, A ; G) & \stackrel{f^{p}}{\leftarrow} & H^{p}(Y, B ; G) \\
\delta^{p} \uparrow & & \uparrow \delta^{p} \\
H^{p-1}(A ; G) & \stackrel{f^{p} \mid}{\leftarrow} & H^{p-1}(B ; G)
\end{array}
$$

(4) Dadas as inclusões $i:(A, \emptyset) \rightarrow(X, \emptyset)$ e $j:(X, \emptyset) \rightarrow(X, A)$, então a sequência abaixo é exata,

$$
\ldots \leftarrow H^{p}(A ; G) \stackrel{i^{*}}{\leftarrow} H^{p}(X ; G) \stackrel{j^{*}}{\leftarrow} H^{p}(X, A ; G) \stackrel{\delta^{*}}{\leftarrow} H^{p-1}(A ; G) \leftarrow \ldots
$$

(5) Se $h \simeq k$ (homotópicas), então $h^{p}=k^{p}$, para todo inteiro p;

(6) Sejam $(X, A)$ e $U \subset X$ aberto tal que $\bar{U} \subset \operatorname{int}(A)$. Então, a inclusão $j:(X-U, A-U) \rightarrow(X, A)$ induz um isomorfismo:

$$
j^{*}: H^{p}(X, A ; G) \rightarrow H^{p}(X-U, A-U ; G)
$$

(7) Se $X=\{x\}$ então $H^{0}(X ; G)=G$ e $H^{p}(X ; G)=0$, para $p \neq 0$.

\section{Cohomologia Singular}

Seja $A$ um anel comutativo com unidade.

Definição 1.4.1. Um complexo de cocadeias é uma sequência $C=\left(C^{p}, \delta^{p}\right), p \geq 0$, de A-módulos $C^{p}$ e homomorfismos $\delta^{p}: C^{p} \rightarrow C^{p+1}$ tais que $\delta^{p+1} \circ \delta^{p}=0$.

Definição 1.4.2. (1) Cada elemento $u \in C^{p}$ é chamado cocadeia de dimensão p;

(2) Se $\delta^{p} u=0$ então u é chamado cociclo de dimensão $p$;

(3) $Z^{p}=Z^{p}(C)=K e r \delta^{p}$ é o conjunto de todos os cociclos de dimensão $p$;

(4) $B^{p}=B^{p}(C)=I m \delta^{p-1}$ é o conjunto de todas as cocadeias de dimensão $p$.

Definição 1.4.3. O grupo de cohomologia de dimensão $p$ do complexo $C$ é definido por:

$$
H^{p}(C)=\frac{Z^{p}}{B^{p}}
$$


Exemplo 1.4.4. Dado um complexo de cadeias $\left(C_{p}, \partial_{p}\right)$.

Considere, $C^{p}=\operatorname{Hom}\left(C_{p} ; A\right)=\left\{h: C_{p} \rightarrow A ; h\right.$ é homomorfismo $\}$ e $\delta^{p}: C^{p} \rightarrow C^{p+1}$ definido por

$$
\begin{aligned}
& \delta^{p}: C^{p} \quad \rightarrow \quad C^{p+1} \\
& u \mapsto \delta^{p}(u): C_{p+1} \rightarrow A \\
& x \quad \mapsto \quad\left(\delta^{p}(u)\right)(x)=u\left(\partial_{p}(x)\right)
\end{aligned}
$$

Obtemos assim, $\left(C^{p}, \delta^{p}\right)$ um complexo de cocadeias.

Observação 1.4.5. Portanto, a partir de uma homologia podemos definir uma cohomologia.

Definição 1.4.6. Dados $A, B$ grupos abelianos. Um homomorfismo $f: A \rightarrow B$ induz um homomorfismo dual

$$
\begin{array}{cl}
\tilde{f}: \operatorname{Hom}(B ; G) & \rightarrow \operatorname{Hom}(A ; G) \\
(\phi: B \rightarrow G) & \mapsto \tilde{f}(\phi)=\phi \circ f
\end{array}
$$

Exemplo 1.4.7. Seja a sequência exata de grupos

$$
0 \rightarrow \mathbb{Z} \stackrel{f}{\rightarrow} \mathbb{Z} \stackrel{g}{\rightarrow} \mathbb{Z}_{2} \rightarrow 0
$$

onde $f(n)=2 n$ e $g(n)= \begin{cases}0 ; & \text { se } n \text { é par } \\ 1, & \text { se } n \text { é ímpar }\end{cases}$

Então o dual da sequência é

$$
0 \rightarrow \operatorname{Hom}\left(\mathbb{Z}_{2} ; \mathbb{Z}\right) \stackrel{g^{*}}{\rightarrow} \operatorname{Hom}(\mathbb{Z} ; \mathbb{Z}) \stackrel{f^{*}}{\rightarrow} \operatorname{Hom}(\mathbb{Z} ; \mathbb{Z}) \rightarrow 0
$$

Temos $\operatorname{Hom}\left(\mathbb{Z}_{2} ; \mathbb{Z}\right)=0$ e $\operatorname{Hom}(\mathbb{Z} ; \mathbb{Z})$ é isomorfo a $\mathbb{Z}$, pois é grupo cíclico infinito. Agora, como $f$ é a multiplicação por 2 , então $f^{*}$ também será. Assim, $f^{*}$ não é sobrejetora, consequentemente, o dual da sequência não é exata.

Observação 1.4.8. Isto mostra que nem sempre o dual de uma sequência exata é exata.

O teorema a seguir nos dá uma condição necessária para que o dual de uma sequência exata seja exata. 
Teorema 1.4.9 ([21], Teorema 41.2). Dados uma sequência exata curta de A-módulos

$$
0 \rightarrow X^{\prime} \rightarrow X \rightarrow X^{\prime \prime} \rightarrow 0
$$

e um A-módulo $Y$, tal que a sequência acima cinde. Então,

$$
0 \rightarrow \operatorname{Hom}\left(X^{\prime \prime}, Y\right) \rightarrow \operatorname{Hom}(X, Y) \rightarrow \operatorname{Hom}\left(X^{\prime}, Y\right) \rightarrow 0
$$

é exata.

Dados $X$ um espaço topológico e $R$ um anel comutativo com unidade. Seja $S_{r}(X)$ o $R$-módulo livre das cadeias de dimensão $r$, ou seja,

$$
S_{r}(X)=\left\{\sum_{\sigma} x_{\sigma} \sigma ; \sigma \text { é um } r \text {-simplexo singular e } x_{\sigma} \in R\right\} .
$$

Dessa forma, podemos considerar

$$
S^{r}(X)=\operatorname{Hom}\left(S_{r}(X) ; R\right)=\left\{u: S_{r}(X) \rightarrow R ; u \text { é homomorfismo }\right\} .
$$

Além disso, definimos $\delta: S^{r}(X) \rightarrow S^{r+1}(X)$, por $(\delta u)(x)=u(\partial x)$. Com isso, obtemos que $\delta \circ \delta=0$.

Definição 1.4.10. A cohomologia singular de $X$ com coeficientes em $R$ é definida por:

$$
H^{r}(X)=\frac{\operatorname{Ker}\left(\delta^{r}\right)}{\operatorname{Im}\left(\delta^{r-1}\right)}
$$

Definição 1.4.11. Dada $f: X \rightarrow Y$ contínua e a aplicação de cadeias correspondente $f_{\#}: S_{p}(X ; R) \rightarrow S_{p}(Y ; R)$. O dual de $f_{\#}$ é denotado por $f^{\#}$ e é tal que

$$
\begin{aligned}
f^{\#}: S^{p}(Y ; R) & \rightarrow S^{p}(X ; R) \\
\varphi^{p} & \mapsto\left(f^{\#}\left(\varphi^{p}\right)\right)\left(\sigma_{p}\right)=\varphi^{p}\left(f_{\#}\left(\sigma_{p}\right)\right)
\end{aligned}
$$

Definição 1.4.12. Dada $f: X \rightarrow Y$, temos que $f$ induz um homomorfismo em nível de cohomologia: 


$$
\begin{aligned}
f^{*}: H^{p}(Y) & \rightarrow H^{p}(X) \\
u^{p} & \mapsto\left(f^{*}\left(u^{p}\right)\right)\left(v_{p}\right)=u^{p}\left(f_{*}\left(v_{p}\right)\right)
\end{aligned}
$$

\section{Produto Cap}

O produto cap é uma família de $R$-funções lineares:

$$
\begin{aligned}
H^{k}(X, A ; M) \otimes H_{n}(X, A \cup B ; N) & \rightarrow H_{n-k}\left(X, B ; M \otimes_{R} N\right) \\
x \otimes y & \mapsto x \cap y
\end{aligned}
$$

$M, N$ são $R$-módulos e $(A, B)$ um par excisivo em $X$.

Em geral, usa-se $M=N=M \otimes N=R$.

Primeiro define-se o produto cap em nível de cadeias e cocadeias e depois podemos estendê-lo por linearidade.

$$
\begin{array}{cl}
S^{p}(X ; R) \otimes S_{p+q}(X ; R) & \rightarrow S_{q}(X ; R) \\
\varphi^{p} \otimes c_{p+q} & \mapsto \varphi^{p} \cap c_{p+q}
\end{array}
$$

$\operatorname{Dados} \varphi^{p} \in S^{p}(X ; R), c_{p+q}: \Delta_{p+q}=\left[e_{0}, \ldots, e_{p+q}\right] \rightarrow X$ e $b \in R$, definimos,

$$
\varphi^{p} \cap\left(c_{p+q} \otimes b\right)=\left.\varphi^{p}\left(\left.c_{p+q}\right|_{\left[e_{q}, \ldots, e_{p+q}\right]}\right) \cdot c_{p+q}\right|_{\left[e_{0}, \ldots, e_{q}\right]} \otimes b .
$$

Propriedades:

1. Seja $f: X \rightarrow Y$ contínua, $\varphi^{p} \in S^{p}(Y ; R)$ e $c_{p+q} \in S_{p+q}(X ; R)$. Então,

$$
f_{\#}\left(f^{\#}\left(\varphi^{p}\right) \cap c_{p+q}\right)=\varphi^{p} \cap f_{\#} c_{p+q}
$$

De fato,

$$
\begin{gathered}
f_{\#} c_{p+q} \cap \varphi^{p}=\left.\varphi^{p}\left(\left.f_{\#} c_{p+q}\right|_{\left[e_{q}, \cdots, e_{p+q}\right]}\right) \cdot f_{\#} c_{p+q}\right|_{\left[e_{0}, \cdots, e_{q}\right]}= \\
=f_{\#}\left(\left.\varphi^{p}\left(\left.f_{\#} c_{p+q}\right|_{\left[e_{q}, \cdots, e_{p+q}\right]}\right) \cdot c_{p+q}\right|_{\left[e_{0}, \cdots, e_{q}\right]}\right)=f_{\#}\left(\left.\left.f^{\#}\left(\varphi^{p}\right) c_{p+q}\right|_{\left[e_{q}, \cdots, e_{p+q}\right]} \cdot c_{p+q}\right|_{\left[e_{0}, \cdots, e_{q}\right]}\right)= \\
f_{\#}\left(f^{\#}\left(\varphi^{p}\right) \cap c_{p+q}\right) .
\end{gathered}
$$


Temos o seguinte diagrama, o qual auxilia a compreensão da propriedade 1.

$$
\begin{array}{ccccc}
S^{p}(X ; R) & \otimes & S_{p+q}(X ; R) & \rightarrow & S_{q}(X ; R) \\
\uparrow f^{\#} & \downarrow f_{\#} & & \downarrow f_{\#} \\
S^{p}(Y ; R) & \otimes & S_{p+q}(Y ; R) & \stackrel{\neg}{\rightarrow} & S_{q}(Y ; R)
\end{array}
$$

2. $\partial\left(\varphi^{p} \cap c_{p+q}\right)=(-1)^{q} \delta \varphi^{p} \cap c_{p+q}+\varphi^{p} \cap \partial c_{p+q}$

Agora, usando a propriedade 2, podemos definir o produto cap em nível de homologias e cohomologias, da seguinte forma:

$$
\begin{aligned}
H^{p}(X ; R) \otimes H_{p+q}(X ; R) & \rightarrow H_{q}(X ; R) \\
{\left[c^{p}\right] \otimes\left[z_{p+q}\right] } & \mapsto\left[c^{p}\right] \cap\left[z_{p+q}\right]=\left[c^{p} \cap z_{p+q}\right]
\end{aligned}
$$

De fato, sejam $\left[c^{p}\right] \in H^{p}(X ; R)$ e $\left[z_{p+q}\right] \in H_{p+q}(X ; R)$ então $\delta^{p}\left(c^{p}\right)=0$ e $\partial_{p+q}\left(z_{p+q}\right)=0$. Logo,

$$
\partial_{q}\left(c^{p} \cap z_{p+q}\right)=(-1)^{q}\left(\delta^{p}\left(c^{p}\right) \cap z_{p+q}\right)+\left(c^{p} \cap \partial_{p+q}\left(z_{p+q}\right)\right)=0 .
$$

Portanto, $c^{p} \cap z_{p+q} \in Z_{q}(X ; R)$, logo representa uma classe de homologia em $H_{q}(X ; R)$.

\section{Produto Cap Relativo}

Definiremos nessa seção o produto cap relativo, este conceito será utilizado nas definições de obstrução de Euler e de obstrução de Euler de uma função. Nos restringimos a uma breve introdução deste assunto, para maiores detalhes sugerimos [21].

Para cada subespaço $A \subset X$, sejam $S^{p}(X ; A ; R) \subset S^{p}(X ; R)$ e $S_{p+q}(A ; R) \subset S_{p+q}(X ; R)$, podemos considerar:

$$
\begin{aligned}
S^{p}(X ; A ; R) \otimes S_{p+q}(A ; R) \subset S^{p}(X ; R) \otimes S_{p+q}(X ; R) & \stackrel{\cap}{\rightarrow} S_{q}(X ; R) \\
\left(\varphi^{p}, c_{p+q}\right) & \mapsto \varphi^{p} \cap c_{p+q}
\end{aligned}
$$

Então para $\varphi^{p} \in S^{p}(X ; A ; R)$ e $c_{p+q} \in S_{p+q}(A ; R)$ temos:

$$
\varphi^{p} \cap c_{p+q}:=\left.\varphi^{p}\left(\left.c_{p+q}\right|_{\left[e_{q}, \cdots, e_{p+q}\right]}\right) \cdot c_{p+q}\right|_{\left[e_{0}, \cdots, e_{q}\right]} \in S_{q}(X ; R) .
$$


Porém, $S^{p}(X ; A ; R)=\left\{\varphi^{p} \in S^{p}(X ; R) ; \varphi^{p}\left(S_{p}(A ; R)\right)=0\right\}$ e $\left.c_{p+q}\right|_{\left[e_{q}, \cdots, e_{p+q}\right]}$ é um $p$-simplexo singular de $A$, logo, $\varphi^{p}\left(\left.c_{p+q}\right|_{\left[e_{q}, \cdots, e_{p+q}\right]}\right)=0$.

Portanto,

$$
\begin{array}{cl}
S^{p}(X ; A ; R) \otimes S_{p+q}(A ; R) & \stackrel{\cap}{\rightarrow} S_{q}(X ; R) \\
\left(\varphi^{p}, c_{p+q}\right) & \mapsto \varphi^{p} \cap c_{p+q}=0
\end{array}
$$

Assim, temos o produto bem definido:

$$
\begin{array}{rlc}
S^{p}(X ; A ; R) \otimes S_{p+q}(X ; A ; R) & \stackrel{\cap}{\rightarrow} & S_{q}(X ; R) \\
\left(\varphi^{p}, c_{p+q}+S_{p+q}(A ; R)\right) & \mapsto & \varphi^{p} \cap\left(c_{p+q}+S_{p+q}(A ; R)\right)
\end{array}
$$

onde, $\varphi^{p} \cap\left(c_{p+q}+S_{p+q}(A ; R)\right):=\varphi^{p} \cap\left[c_{p+q}\right]=\varphi^{p} \cap c_{p+q}$.

O produto acima está bem definido.

De fato, se $\left[c_{p+q}\right]=c_{p+q}+S_{p+q}(A ; R)=c_{p+q}^{\prime}+S_{p+q}(A ; R)=\left[c_{p+q}^{\prime}\right]$ então,

$$
\begin{gathered}
c_{p+q}-c_{p+q}^{\prime} \in S_{p+q}(A ; R) \Rightarrow\left(c_{p+q}-c_{p+q}^{\prime}\right) \cap \varphi^{p}=0, \forall p \\
\Rightarrow \varphi^{p} \cap c_{p+q}-\varphi^{p} \cap c_{p+q}^{\prime}=0 \Rightarrow \varphi^{p} \cap c_{p+q}=\varphi^{p} \cap c_{p+q}^{\prime} \\
\Rightarrow \varphi^{p} \cap\left[c_{p+q}\right]=\varphi^{p} \cap\left[c_{p+q}^{\prime}\right] .
\end{gathered}
$$

E este produto induz um produto cap:

$$
H^{p}(X ; A ; R) \otimes H_{p+q}(X ; A ; R) \stackrel{\cap}{\rightarrow} H_{q}(X ; R)
$$

definido por

$$
\begin{aligned}
c^{p}+B^{p}(X ; A ; R) \cap\left[z_{p+q}\right]+B_{p+q}(X ; A ; R):=\left(c^{p} \cap\left[z_{p+q}\right]\right)+B_{q}(X ; R)= \\
=c^{p} \cap z_{p+q}+B_{q}(X ; R)=\left[c^{p} \cap z_{p+q}\right] .
\end{aligned}
$$




\subsection{Teoria de singularidades}

Nesta seção, apresentaremos as definições e os principais resultados da teoria de singularidades que serão usados para o desenvolvimento deste trabalho. Como referência para este assunto, recomendamos [9, 18, 26].

Um dos nossos objetivos é estudar localmente funções analíticas $f: \mathbb{C}^{n} \rightarrow \mathbb{C}$. Para isso, introduziremos uma relação de equivalência no espaço das funções analíticas definidas em um aberto contendo a origem de $\mathbb{C}^{n}$, da seguinte forma:

Definição 1.5.1. Dizemos que a função $f: \mathbb{C}^{n} \rightarrow \mathbb{C}$ é equivalente à função $g: \mathbb{C}^{n} \rightarrow \mathbb{C}$, se existe um aberto $U \subset \mathbb{C}^{n}$, com $0 \in U$, tal que $f_{\mid U} \equiv g_{\mid U}$. As classes de equivalência são chamadas de germes, denotadas por $f:\left(\mathbb{C}^{n}, 0\right) \rightarrow \mathbb{C}$, ou simplesmente, por $f$. A coleção de todos esses germes de funções citados acima é denotada por $\mathcal{O}_{n}$. Observemos que $\mathcal{O}_{n}$ é um anel noetheriano local cujo ideal maximal é dado por $\mathcal{M}_{n}=\left\{f \in \mathcal{O}_{n}: f(0)=0\right\}$.

Teorema 1.5.2 ([18], Teorema 2.10). Sejam $f:\left(\mathbb{C}^{n}, 0\right) \rightarrow(\mathbb{C}, 0)$ germe de função analítica, $V=f^{-1}(0), \mathbb{B}_{\varepsilon}$ bola centrada na origem e de raio $\varepsilon$ e $K=V \cap \mathbb{S}^{2 n-1}$. Se $\varepsilon$ for suficientemente pequeno temos que $V \cap \mathbb{B}_{\varepsilon}$ é homeomorfo ao cone $C(K)=\{t z: 0 \leq t \leq 1, z \in K\}$.

Seja $\phi: \mathbb{S}_{\varepsilon} \backslash K \rightarrow \mathbb{S}^{1}$ dada por $\phi(z)=\frac{f(z)}{|f(z)|}$, e denotemos $\phi^{-1}\left(e^{i \theta}\right)=F_{\theta}$.

O resultado básico da teoria de Milnor é o Teorema da Fibração, que mostra que $\phi$ define uma fibração de $\mathbb{S}_{\varepsilon} \backslash K \rightarrow \mathbb{S}^{1}$ com fibra $F_{\theta}$.

Teorema 1.5.3 ([18], Teorema 4.8). Para $\varepsilon$ suficientemente pequeno temos que $\mathbb{S}_{\varepsilon} \backslash K$ é um fibrado diferenciável localmente trivial sobre $\mathbb{S}^{1}$, com projeção $\phi$ e fibra $F_{\theta}$ como acima.

Definição 1.5.4. Fo é dita a fibra de Milnor de $f$.

Em [18], J. Milnor provou que as fibras $F_{\theta}$ são todas difeomorfas, isto segue do fato que a base $\mathbb{S}^{1}$ é uma variedade conexa. Também em [18], J. Milnor apresentou uma descrição alternativa da fibra de Milnor.

Teorema 1.5.5 ([18], Teorema 5.11). Sejam $c \in \mathbb{C}^{*}$ um número complexo suficientemente próximo de zero e $f:\left(\mathbb{C}^{n}, 0\right) \rightarrow(\mathbb{C}, 0)$ germe de aplicação analítica, então a interseção 
da hipersuperfície complexa $f^{-1}(c)$ com a bola aberta $\mathbb{B}_{\varepsilon}(0)$ de centro 0 e raio $\varepsilon$ de $\mathbb{C}^{n}$ é uma variedade suave difeomorfa a fibra $F_{\theta}$.

Nas hipóteses do teorema acima, denotaremos $f^{-1}(c) \cap \mathbb{B}_{\varepsilon}(0)=F_{f}$.

O número de Milnor, que definiremos a seguir, é um invariante de suma importância na teoria de singularidades, auxiliando na teoria de classificação. Para este conceito e resultados a ele relacionados sugerimos [8] e [18].

Teorema 1.5.6 ([18], Teorema 6.5). Seja $f \in \mathcal{O}_{n}$ germe de função analítica com singularidade isolada na origem, então cada fibra $F_{f}$ tem o mesmo tipo de homotopia de um buquê de esferas $\mathbb{S}^{n-1}$.

Definição 1.5.7. Sejam $f \in \mathcal{O}_{n}$, germe de função analítica com singularidade isolada na origem. Definimos o número de Milnor $\mu(f)$ como o número de esferas do buquê dado pelo Teorema anterior.

Teorema 1.5.8. Sejam $f \in \mathcal{O}_{n}$, germe de função analítica com singularidade isolada na origem e $J(f)$ o ideal jacobiano de $f$. Então,

$$
\mu(f)=\operatorname{dim}_{\mathbb{C}} \frac{\mathcal{O}_{n}}{J(f)}
$$

\subsection{Variedades algébricas e espaços analíticos complexos}

Nesta seção trabalharemos com o caso complexo.

\section{Variedades algébricas}

O $n$-espaço afim sobre $\mathbb{C}$, denotado por $\mathbb{A}^{n}$ é o conjunto de todas as $n$-uplas de elementos de $\mathbb{C}$. Um ponto $p \in \mathbb{A}^{n}$ é dado por $p=\left(a_{1}, \ldots, a_{n}\right)$, com $a_{i} \in \mathbb{C}$, e $a_{i}$ a $i$-ésima coordenada de $p$.

Seja $\mathbb{C}\left[x_{1}, \cdots, x_{n}\right]$ o anel de polinômios em $n$ variáveis sobre $\mathbb{C}$. Podemos interpretar os elementos de $\mathbb{C}\left[x_{1}, \cdots, x_{n}\right]$ como funções do espaço afim $\mathbb{A}^{n}$ sobre $\mathbb{C}$, com $f(p)=f\left(a_{1}, \ldots, a_{n}\right)$, onde $f \in \mathbb{C}\left[x_{1}, \cdots, x_{n}\right]$ e $p \in \mathbb{A}^{n}$. Desta forma, faz sentido falar no conjunto de zeros de $f$, que denotaremos por $Z(f)=\left\{p \in \mathbb{A}^{n} ; f(p)=0\right\}$. De 
uma forma mais geral, se $T$ for um subconjunto de $\mathbb{C}\left[x_{1}, \cdots, x_{n}\right]$, podemos definir o conjunto de zeros de $T$ como sendo o conjunto de zeros comuns a todos os elementos de $T$. Denotaremos esse conjunto da seguinte forma,

$$
Z(T)=\left\{p \in \mathbb{A}^{n} ; f(p)=0 \forall f \in T\right\}
$$

Se $I$ for o ideal de $\mathbb{C}\left[x_{1}, \cdots, x_{n}\right]$ gerado por $T$ então $Z(T)=Z(I)$. Mais ainda, como $\mathbb{C}\left[x_{1}, \cdots, x_{n}\right]$ é um anel Noetheriano, pelo Teorema da base de Hilbert qualquer ideal $I$ tem um número finito de geradores $f_{1}, \ldots, f_{r}, \operatorname{logo} Z(T)$ pode se expressar como o conjunto de zeros comuns de um número finito de polinômios $f_{1}, \ldots, f_{r}$.

Definição 1.6.1. Um subconjunto $Y$ de $\mathbb{A}^{n}$ é chamado conjunto algébrico, se existe um subconjunto $T \subset \mathbb{C}\left[x_{1}, \cdots, x_{n}\right]$ tal que $Y=Z(T)$.

Proposição 1.6.2 ([13], Proposição 1.11). A união de dois conjuntos algébricos é um conjunto algébrico. A intersecção de uma família arbitrária de conjuntos algébricos também é um conjunto algébrico.

Para a demonstração deste resultado e referência básica para o assunto ver [13].

Definição 1.6.3. Os conjuntos algébricos são os conjuntos fechados de uma topologia de $\mathbb{A}^{n}$, denominada topologia de Zariski.

Definição 1.6.4. Dizemos que um subconjunto não vazio $Y$ contido em $X$ é irredutível, se o mesmo não pode se expressar como uma união $Y=Y_{1} \cup Y_{2}$, onde $Y_{1}, Y_{2}$ são fechados em $Y$.

Definição 1.6.5. Uma variedade algébrica afim é um subconjunto irredutivel de $\mathbb{A}^{n}$ (com a topologia induzida).

\section{Espaços analíticos complexos}

Analogamente ao caso de polinômios, podemos também estudar o conjunto de zeros de uma ou mais funções analíticas. Estes são os chamados espaços analíticos. Nesta seção, trabalharemos com $\mathbb{C}^{n}$ dotado da topologia usual. 
Definição 1.6.6. Seja $U$ um aberto de $\mathbb{C}^{n}$ e seja $X$ um subconjunto de $U$. Dizemos que $X$ é um subconjunto analítico de $U$ se para todo $a \in U$, existem um domínio $V$ tal que $a \in V \subset U$ e funções analíticas $f_{1}, \ldots, f_{r}$ em $V$ tais que:

$$
X \cap V=\left\{z \in V ; f_{1}(z)=\ldots=f_{r}(z)=0\right\} .
$$

Dizemos que $X \subset \mathbb{C}^{n}$ é um conjunto analítico, se $X$ é um subconjunto analítico de um aberto de $\mathbb{C}^{n}$.

Dizemos que $X \subset \mathbb{C}^{n}$ é um conjunto analítico em $z \in \mathbb{C}^{n}$, se existe um dominio $U \subset \mathbb{C}^{n}$ tal que $z \in U$ e $X \cap U$ é um subconjunto analítico de $U$. Observemos que $X$ é um conjunto analítico se ele é um conjunto analítico em cada $z \in X$.

Definição 1.6.7. Seja $X$ um conjunto analítico, dizemos que um ponto z de $X$ é um ponto regular ou suave, se para alguma vizinhança $U$ de $z$, o conjunto $U \cap X$ pode ser descrito como o conjunto dos zeros de um número finito de funções analíticas em $U$ que possuem z como ponto regular. Um ponto de $X$ não regular é chamado de ponto singular de $X$.

Agora consideremos a seguinte definição:

Definição 1.6.8. Consideremos o conjunto de pares $\left(V_{\alpha}, U_{\alpha}\right)$, onde $U_{\alpha}$ é uma vizinhança aberta da origem em $\mathbb{C}^{n}$ e $V_{\alpha}$ subconjuntos de $U_{\alpha}$. Dois tais pares $\left(V_{1}, U_{1}\right)$ e $\left(V_{2}, U_{2}\right)$ são equivalentes se existe uma vizinhança da origem $W \subset U_{1} \cap U_{2}$ tal que $V_{1} \cap W=V_{2} \cap W$. Uma classe de equivalência destes pares é chamada germe na origem em $\mathbb{C}^{n}$.

Se $f \in \mathcal{O}_{n}$, a classe de equivalência do conjunto $Z(f)=\{x: f(x)=0\}$, onde $f$ é um representante do germe $f$, é denotada por $\mathcal{V}(f)$; se $f_{1}$ e $f_{2}$ são dois representantes de um mesmo germe, então os conjuntos $\mathcal{V}\left(f_{1}\right)$ e $\mathcal{V}\left(f_{2}\right)$ são iguais.

Definição 1.6.9. Um germe de espaço analítico $(X, 0)$ em torno da origem é o germe do subconjunto

$$
X=Z\left(f_{1}\right) \cap \ldots \cap Z\left(f_{r}\right)=Z\left(f_{1}, \ldots, f_{r}\right),
$$

$\operatorname{para} f_{1}, \ldots, f_{r} \in \mathcal{O}_{n}$ 
Definimos o ideal de um germe de espaço analítico $X$ por

$$
\mathcal{I}(X)=\left\{f \in \mathcal{O}_{n}: X \subset f^{-1}(0)\right\}
$$

Definição 1.6.10. Dizemos que um germe de espaço analítico $X$ é irredutível, quando para quaisquer germes $X_{1}$ e $X_{2}$ tais que $X=X_{1} \cup X_{2}$ então $X=X_{1}$ ou $X=X_{2}$. Neste caso, dizemos que $X$ é uma variedade analítica.

O nosso objetivo é estudar a natureza de tais espaços analíticos na vizinhança de algum ponto fixado em $\mathbb{C}^{n}$, o qual sem perda de generalidade consideraremos a origem.

Proposição 1.6.11. Seja $X$ um germe de espaço analítico, então existem um inteiro positivo $p$ e $X_{1}, \ldots, X_{p}$ variedades irredutiveis, com $X_{i}$ não contida em $X_{j}$, para todo $i \neq j$, tais que $X=X_{1} \cup \ldots \cup X_{p}$. Essas variedades são unicamente determinadas, a menos da ordem, e são chamadas de componentes irredutiveis de $X$.

Definição 1.6.12. A dimensão de um germe de espaço analítico é a maior das dimensões de suas componentes irredutiveis.

Definição 1.6.13. Dizemos que um germe de espaço analítico X é equidimensional, quando todas as suas componentes irredutiveis tem a mesma dimensão.

Definição 1.6.14. Dizemos que um germe $(X, 0)$ de $X=Z\left(f_{1}, \ldots, f_{r}\right)$ na origem é reduzido, se a $\mathbb{C}$-álgebra $\frac{\mathcal{O}_{n}}{\left\langle f_{1}, \ldots, f_{r}\right\rangle}$ não possui elementos nilpotentes.

\section{Interseção completa com singularidade isolada}

A definição de número de Milnor que vimos na seção 1.5 foi introduzida por J. Milnor em [18] para hipersuperfícies com singularidade isolada, existe também uma definição mais geral para este invariante, para a qual precisaremos da noção de interseção completa com singularidade isolada, que é um caso particular de variedade analítica.

Seja $f: \mathbb{C}^{n+k} \rightarrow \mathbb{C}^{k}$ uma aplicação holomorfa e consideremos $V=f^{-1}(0)$.

Definição 1.6.15. Dizemos que $V$ é uma interseção completa (geométrica) se $\operatorname{dim}_{\mathbb{C}} V=n$ e se $V$ for definida como o conjunto comum de zeros de $k$ funções holomorfas. 
Também, $V$ é uma interseção completa se o ideal $\mathcal{I}(V)=\left\{g \in \mathcal{O}_{n+k}: V \subset g^{-1}(0)\right\}$ é gerado por $k$ funções holomorfas.

Proposição 1.6.16. Sejam $f: \mathbb{C}^{n+k} \rightarrow \mathbb{C}^{k}$ uma aplicação holomorfa e $V=f^{-1}(0)$. Então, $V$ é uma interseção completa geométrica se, e somente se, é uma interseção completa.

No caso em que $V$ tenha singularidade isolada, ou seja, $V$ seja uma interseção completa com singularidade isolada, abreviaremos por ICSI. Neste caso, podemos generalizar o número de Milnor, esta definição foi dada em [12] por H. Hamm.

Teorema 1.6.17 ([12]). Sejam $f=\left(f_{1}, \ldots, f_{k}\right): \mathbb{C}^{n+k} \rightarrow \mathbb{C}^{k}$ uma aplicação (germe) analítica, $V=f^{-1}(0)$ uma ICSI e $c \in \mathbb{C}^{k}$ suficientemente próximo de $0 \in \mathbb{C}^{k}$. Então, cada fibra $F_{f}=f^{-1}(c) \cap \mathbb{B}_{\varepsilon}(0)$ tem o mesmo tipo de homotopia de um buquê de esferas $\mathbb{S}^{n}$.

Definição 1.6.18. Sejam $f=\left(f_{1}, \ldots, f_{k}\right): \mathbb{C}^{n+k} \rightarrow \mathbb{C}^{k}$ uma aplicação (germe) analítica e $V=f^{-1}(0)$ uma ICSI na origem. Dada uma fibra $F_{f}$, definimos o número de Milnor de $V, \mu(V)$, como sendo o número de esferas do buquê citado no teorema anterior.

Teorema 1.6.19 (Fórmula de Lê-Greuel). [[6], Teorema 4.6] Seja uma aplicação analítica $f=\left(f_{1}, \ldots, f_{k}\right): \mathbb{C}^{n+k} \rightarrow \mathbb{C}^{k}$ com uma singularidade isolada na origem. Seja $V_{1}$ um germe na origem de uma ICSI em $\mathbb{C}^{n}$ e $V$ um germe na origem de uma ICSI definida em $V_{1}$ por $f_{k}=0$. Seja $f_{1}, \cdots, f_{k-1}$ um sistema dos geradores do ideal que define $V_{1}$ na origem em $\mathbb{C}^{n}$. Então,

$$
\mu\left(V_{1}\right)+\mu(V)=\operatorname{dim}_{\mathbb{C}} \frac{\mathcal{O}_{n}}{\left(f_{1}, \cdots, f_{k-1}, J\left(f_{1}, \cdots, f_{k}\right)\right)}
$$





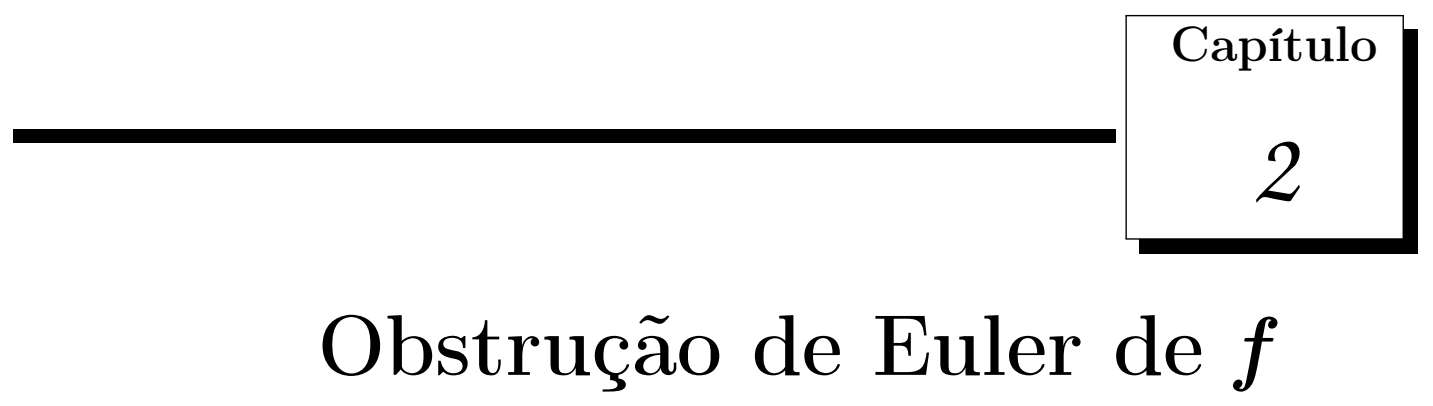

O objetivo deste capítulo é expor conceitos necessários para a definição e estudo da obstrução de Euler e da obstrução de Euler de uma função. Como referência básica para esse assunto sugerimos [3].

\subsection{Estratificação de Whitney}

Mostraremos nesta seção a noção de estratificação de Whitney, introduzida por H. Whitney em [28] e amplamente utilizada desde então. Uma referência para esse assunto é [9].

Definição 2.1.1. Sejam $M$ uma variedade suave e $V \subset M$. Uma estratificação localmente finita de $V$ é uma partição de $V$ em subvariedades de $M$ (chamadas de estratos) tais que, para todo ponto de $V$ existe uma vizinhança em $M$ que encontra apenas um número finito de estratos.

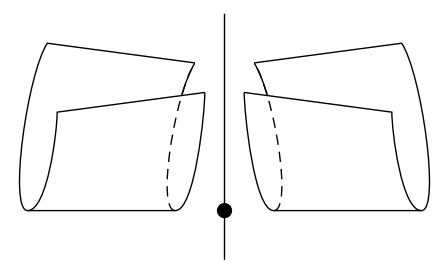


Definição 2.1.2. Dizemos que a estratificação $\left\{V_{\alpha}\right\}$ de $V$ satisfaz a condição de fronteira, se para dois estratos $V_{\alpha}$ e $V_{\beta}$, tais que $V_{\alpha} \cap \bar{V}_{\beta} \neq \emptyset$ então $V_{\alpha} \subset \bar{V}_{\beta}$.

\section{Condições de Whitney}

Definição 2.1.3. A estratificação $\left\{V_{\alpha}\right\}$ satisfaz as condições de Whitney se para todo par $\left(V_{\alpha}, V_{\beta}\right)$ de estratos, tais que $V_{\beta}$ esteja no fecho de $V_{\alpha}$ e para todo ponto y de $V_{\beta}$ temos:

a) Para toda sequência de pontos $\left\{x_{i}\right\}$ de $V_{\alpha}$ convergindo para $y$, tal que o limite

$$
\lim _{i \rightarrow \infty} T_{x_{i}}\left(V_{\alpha}\right)=T
$$

existe na Grassmanniana correspondente, então $T$ contém $T_{y}\left(V_{\beta}\right)$.

b) Se além disso temos uma sequência $\left\{y_{i}\right\}$ de pontos de $V_{\beta}$ com limite y e tal que o limite de direções

$$
\lim _{i \rightarrow \infty} \overline{x_{i} y_{i}}=\lambda
$$

existe no espaço projetivo, então $T$ contém $\lambda$.

Estas são as chamadas condição (a) e condição (b) de Whitney.

H. Whitney mostrou em seu trabalho [28] que toda variedade analítica complexa admite uma estratificação satisfazendo essas duas condições.

Uma estratificação que satisfaz as condições de Whitney e a condição de fronteira é chamada de estratificação de Whitney, ou estratificação Whitney regular.

Exemplo 2.1.4. Consideremos como primeiro exemplo o cone $C$ com vértice na origem, e a estratificação $\left\{V_{1}, V_{2}\right\}$, onde $V_{1}$ é uma geratriz do cone e $V_{2}=C \backslash V_{1}$. Neste caso, as condições $(a)$ e (b) de Whitney não são satisfeitas. Para ver isto, basta considerar uma sequência $\left\{x_{i}\right\}$ de pontos de $C$, situados todos sobre uma geratriz $L$ do cone que não seja $V_{1}$, cujo limite seja a origem, de tal modo que o segmento $\overline{x_{i} y_{i}}$ tenha sempre a mesma direção $\lambda$. 


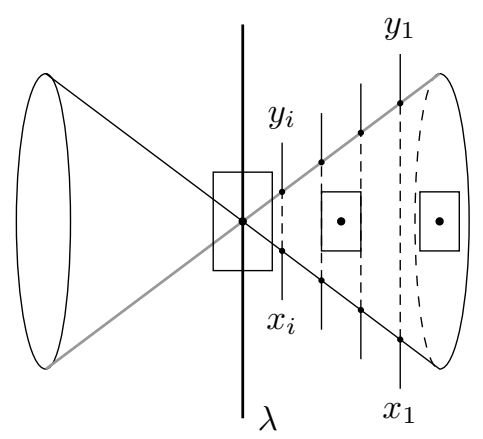

A condição ( $a$ ) não é satisfeita, já que o limite dos espaços tangentes $T_{x_{i}} L$ não contém o espaço $T_{0}\left(V_{1}\right)$, a verificação de que a condição $(b)$ também não é satisfeita vem do fato que $\lambda$ não está contida no limite dos espaços tangentes $T_{x_{i}} L$.

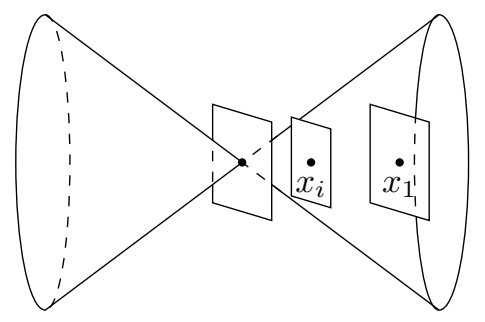

Exemplo 2.1.5. Consideremos agora a variedade $V$ em $\mathbb{C}^{3}$ dada pelo conjunto de zeros de $y^{2}+x^{3}-t^{2} x^{2}=0$. Se tomarmos o eixo da coordenada $t$ como um estrato $V_{1}$ e a parte regular da variedade $V_{\text {reg }}$ como sendo outro estrato, temos que a estratificação $\left\{V_{1}, V_{2}\right\}$ satisfaz a condição $(a)$, mas não a condição $(b)$. Porém se acrescentarmos um estrato de dimensão zero, a origem de $\mathbb{C}^{3}$, teremos as duas condições de Whitney satisfeitas.

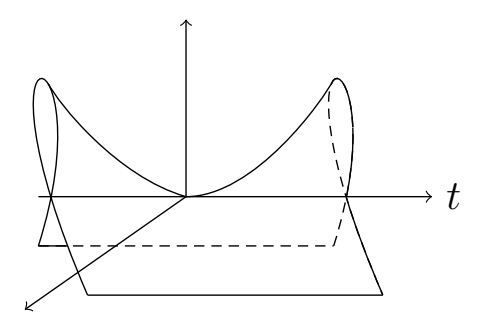

Figura 2.1: Variedade Singular. 


\subsection{Modificação de Nash}

Abordaremos nesta seção, os conceitos de modificação de Nash e de fibrado de Nash. Para resultados sobre modificação de Nash e resolução de singularidades sugerimos [23].

Denotaremos por $G(d, n)$ a Grassmanniana de $d$-planos de $\mathbb{C}^{n}$ (para maiores detalhes sobre a variedade Grassmanniana sugerimos [15]). Seja X um representante de $(X, 0)$ um germe de espaço analítico complexo, equidimensional de dimensão complexa $d$ com $X \subset U$, onde $U$ é um conjunto aberto de $\mathbb{C}^{n}$.

Sobre a parte regular $X_{\text {reg }}$ de $X$, podemos definir $\phi: X_{\text {reg }} \rightarrow U \times G(d, n)$ a aplicação de Gauss da seguinte forma:

$$
\phi(x)=\left(x, T_{x} X_{r e g}\right)
$$

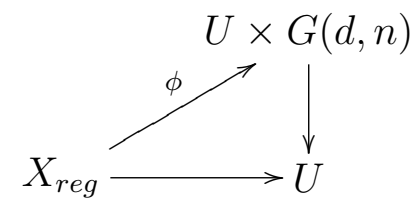

Definição 2.2.1. A modificação ou transformação de Nash $\tilde{X}$ é definida como o fecho da imagem de $\phi$ em $U \times G(d, n)$.

Observação 2.2.2. Como observado em [3] na página 130, $\widetilde{X}$ é um espaço analítico complexo munido de uma projeção analítica natural $\nu: \widetilde{X} \rightarrow X$. Cuja restrição $\left.\nu\right|_{\nu^{-1}\left(X_{r e g}\right)}$ é holomorfa, bijetora e sua inversa também é holomorfa.

Denotemos por $U(d, n)$ o fibrado tautológico sobre $G(d, n)$ o qual contém os elementos da forma, $(P, v)$, onde $P \in G(d, n)$ e $v \in P$. E denotemos por $T$ o fibrado correspondente a extensão trivial de $U(d, n)$ sobre $U \times G(d, n)$, ou seja, $T$ contém os elementos da forma, $(x, P, v)$, onde $(x, P) \in U \times G(d, n)$ e $v \in P$. 
Definição 2.2.3. O fibrado de Nash $\widetilde{T}$ de base $\widetilde{X}$ é a restrição de $T$ sobre $\widetilde{X}$, temos então o diagrama:

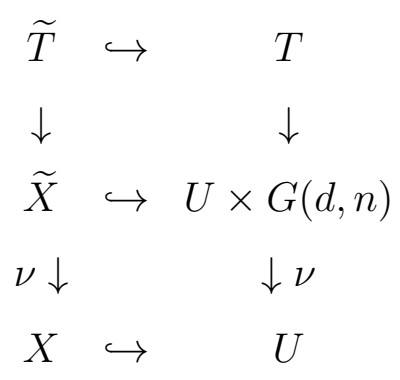

Exemplo 2.2.4. A modificação de Nash do cone $C$ é um cilindro $\widetilde{C}$.

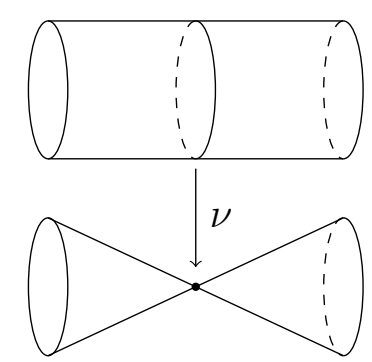

Observação 2.2.5. Uma observação importante é o fato que a modificação de Nash de um conjunto singular nem sempre é regular, ou seja, nem sempre a modificação de Nash resolve a singularidade de uma variedade algébrica ou analítica.

Exemplo 2.2.6. Consideremos, por exemplo, a curva plana formada pela cúspide e uma reta $l$, tal que $l$ seja o limite

$$
\lim _{p \rightarrow 0} l_{p}
$$

onde $l_{p}$ é a reta tangente à cúspide no ponto $p$, esta curva está representada na figura 2.2 no plano cinza.

A modificação de Nash da curva plana é a curva espacial ilustrada na figura 2.2. Note que $(0, l)$ continua sendo um ponto singular na modificação de Nash, já que este continua sendo um ponto de cruzamento entre duas curvas. 


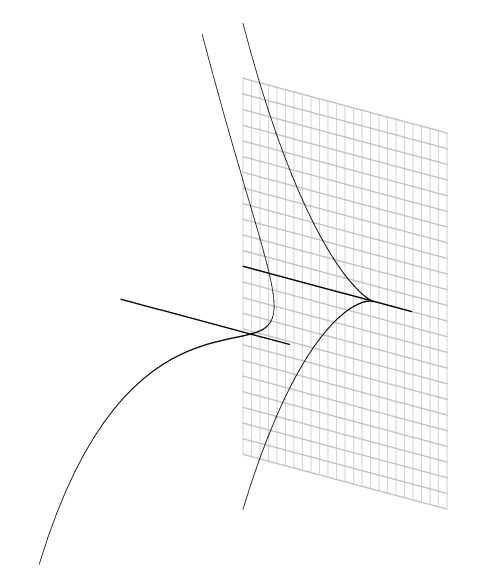

Figura 2.2

\subsection{Obstrução de Euler}

A obstrução de Euler foi definida por R. D. MacPherson em [16]. Apresentaremos nesta seção, uma outra definição dada por J.-P. Brasselet e M.-H. Schwartz em [2], que obtiveram, nesse mesmo artigo, a equivalência entre as duas definições.

$\operatorname{Sejam~}(X, 0)$ germe de espaço analítico complexo, equidimensional e $X \subset U$ um representante do germe, onde $U$ é um subconjunto aberto de $\mathbb{C}^{n}$. Consideremos $\left\{V_{i}\right\}$ uma estratificação de Whitney de $U$ adaptada à $X$, ou seja, $X$ é união de estratos de $U$. Suponhamos que a origem seja um estrato e que o representante $\mathrm{X}$ é suficientemente pequeno para que $\{0\}$ esteja no fecho de todos os estratos. Seja $T U$ o fibrado tangente de $U$.

Definição 2.3.1. Um campo de vetores estratificado $v$ em X é uma seção contínua da restrição $\left.T U\right|_{X}$ tal que se $x \in X \cap V_{i}$ então $v(x) \in T_{x} V_{i}$.

Seja $\mathbb{B}_{\varepsilon}(a)$ a bola em $\mathbb{C}^{n}$ centrada em $a$ e de raio $\varepsilon$. 
Definição 2.3.2. Um campo de vetores estratificado é radial em a $\in X$ se, para cada bola suficientemente pequena $\mathbb{B}_{\varepsilon}(a) \subset U$ e para cada ponto da fronteira $x \in \partial \mathbb{B}_{\varepsilon}(a)$, o vetor $v(x)$ está saindo da bola $\mathbb{B}_{\varepsilon}(a)$. Tal campo de vetores tem necessariamente uma singularidade isolada em a.

Lema 2.3.3 ([2], Prop. 9.1). Um campo de vetores estratificado $v$ não nulo definido sobre um subconjunto $A \subset X$ admite um levantamento canônico $\tilde{v}$ como seção nãa nula de $\widetilde{T}$ sobre $\nu^{-1}(A)$.

Demonstração. Temos o seguinte diagrama :

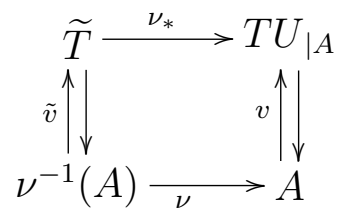

onde $\nu_{*}:\left.\tilde{T} \rightarrow T U\right|_{A}$ é definida por $\nu_{*}(x, P, v(x))=v(x)$.

Seja $x \in A \subset X$.

Para $x \in X_{\text {reg }}$, temos $\nu^{-1}(x)=\left(x, T_{x} X_{\text {reg }}\right)=\tilde{x}$. Como $v(x) \in T_{x} X_{\text {reg }}$, então $\left(x, T_{x} X_{\text {reg }}, v\right) \in \tilde{T}$. Portanto, existe um único vetor $\tilde{v}(\tilde{x})=\left(x, T_{x} X_{\text {reg }}, v\right)$ em $\tilde{T}$ e tal que $\nu_{*}(\tilde{v}(\tilde{x}))=v(x)$.

Agora, consideraremos o caso em que $x$ é um ponto singular de $X$. Temos que $x \in V_{i}$, para algum estrato $V_{i}$ e como o campo de vetores é estratificado, segue que $v(x) \in T_{x} V_{i}$.

Para cada $\tilde{x}$ de $\nu^{-1}(x)$, existe uma sequência $\left\{\tilde{x}_{m}\right\}$ em $\tilde{X}$ tal que $\nu\left(\tilde{x}_{m}\right)=x_{m} \in X_{\text {reg }}$ $\operatorname{com} \lim \tilde{x}_{m}=\tilde{x}$.

Logo, $\tilde{x}_{m}=\left(x_{m}, T_{x_{m}} X_{r e g}\right)$, então o limite

$$
\lim T_{x_{m}} X_{r e g}=P
$$

existe. Assim, pela condição $(a)$ de Whitney, temos $T_{x} V_{i} \subset P, \operatorname{logo} v(x) \in P$. Portanto, temos um elemento bem determinado $\tilde{v}(\tilde{x})=(x, P, v(x)) \in \tilde{T}$, tal que $\nu_{*}(\tilde{v}(\tilde{x}))=v(x)$.

Teorema 2.3.4 (Bertini-Sard, ver [27]). Se $\left\{V_{i}\right\}$ é uma estratificação de Whitney de $X$, então para $\varepsilon>0$ suficientemente pequeno, a esfera $\partial \mathbb{B}_{\varepsilon}(0)$ é transversal aos estratos $V_{i}$. 
A definição a seguir é atribuída à J.-P. Brasselet e M.-H. Schwartz ([2], Prop. 10.1).

Definição 2.3.5. Seja $v$ um campo de vetores radial em $a \in X$ sobre $X \cap \partial \mathbb{B}_{\varepsilon}(a)$ e v $o$ levantamento de $v$ sobre o conjunto $\nu^{-1}\left(X \cap \partial \mathbb{B}_{\varepsilon}(a)\right)$ como uma seção de $\tilde{T}$. A obstrução de Euler $E u_{X}(a)$ é a obstrução para se estender $\tilde{v}$ como uma seção não-nula de $\tilde{T}$ sobre $\nu^{-1}\left(X \cap \mathbb{B}_{\varepsilon}(a)\right)$.

Mais precisamente, o campo $\tilde{v}$ define um cociclo de obstrução $\mathcal{O}(\tilde{v})$, que mede a obstrução para estender $\tilde{v}$ como seção não-nula de $\widetilde{T}$ sobre $\nu^{-1}\left(X \cap \mathbb{B}_{\varepsilon}(a)\right)$ :

$$
\mathcal{O}(\tilde{v}) \in Z^{2 d}\left(\nu^{-1}\left(X \cap \mathbb{B}_{\varepsilon}(a)\right), \nu^{-1}\left(X \cap \partial \mathbb{B}_{\varepsilon}(a)\right)\right)
$$

A obstrução de Euler $E u_{X}(a)$ é o inteiro dado pela avaliação do cociclo $\mathcal{O}(\tilde{v})$ sobre a classe fundamental $\left(\nu^{-1}\left(X \cap \mathbb{B}_{\varepsilon}(a)\right), \nu^{-1}\left(X \cap \partial \mathbb{B}_{\varepsilon}(a)\right)\right)$, ou seja,

$$
E u_{X}(a):=\mathcal{O}(\tilde{v}) \cap\left(\nu^{-1}\left(X \cap \mathbb{B}_{\varepsilon}(a)\right), \nu^{-1}\left(X \cap \partial \mathbb{B}_{\varepsilon}(a)\right)\right)
$$

Observação 2.3.6. Algumas propriedades importantes da obstrução de Euler:

- A obstrução de Euler em um ponto regular é igual à 1. Isto é fácil ver usando simplesmente a definição de obstrução de Euler.

- A obstrução de Euler em um ponto de uma curva é exatamente a multiplicidade do ponto sobre a curva [10]. Esta propriedade sairá também diretamente como corolário do Teorema 2.4 .2 .

- Um outro resultado importante sobre a obstrução de Euler, mostrado inicialmente por J.-P. Brasselet e M.-H. Schwartz [2] e depois por vários autores, é que a obstrução de Euler é constante ao longo de cada estrato de uma estratificação Whitney.

- $E u_{X \times Y}(a, b)=E u_{X}(a) \cdot E u_{Y}(b), \forall a \in X, \forall b \in Y$. 


\subsection{Uma Fórmula Topológica}

A obstrução de Euler não é facilmente calculada a partir de sua definição, o que motivou a obtenção de fórmulas que facilitassem seu cálculo. Apresentaremos nesta seção, uma fórmula de natureza topológica para a obstrução de Euler.

Definição 2.4.1. Uma forma linear complexa genérica (com respeito a X) é uma forma linear complexa $\ell: U \rightarrow \mathbb{C}$ tal que $0 \in \ell^{-1}(0)$ e Ker $(\ell)$ é transversal a todos os limites de espaços tangentes $\left\{T_{x_{n}} V_{i}\right\}$, para todo estrato $V_{i}$ e toda sequência $\left\{x_{n}\right\} \subset V_{i}$ convergindo a 0 .

A demonstração do teorema a seguir, o qual apresenta uma fórmula para a obstrução de Euler, encontra-se em [4].

Teorema 2.4.2 ([4], Teo. 3.1). Sejam $(X, 0)$ um germe de variedade analitica complexa $e\left\{V_{i}\right\}$ uma estratificação de Whitney de $X$. Seja $\ell: U \rightarrow \mathbb{C}$ uma forma linear genérica, onde $U$ é uma vizinhança aberta de $0 \mathrm{em} \mathbb{C}^{n}$. Temos então:

$$
E u_{X}(0)=\sum_{i} \chi\left(V_{i} \cap \mathbb{B}_{\varepsilon} \cap \ell^{-1}\left(t_{0}\right)\right) \cdot E u_{X}\left(V_{i}\right)
$$

onde $\mathbb{B}_{\varepsilon}$ é uma pequena bola fechada em torno da origem em $\mathbb{C}^{n}, t_{0} \in \mathbb{C} \backslash\{0\}$ tal que $\left\|t_{0}\right\|<<1$ e $E u_{X}\left(V_{i}\right)$ é a obstrução de Euler de $X$ em qualquer ponto do estrato $V_{i}$.

Exemplo 2.4.3. Consideremos a função $f(x, y, t)=y^{2}-x^{3}-t^{2} x^{2}$ e denotemos $X=$ $f^{-1}(0)$. Consideremos uma estratificação de Whitney de $X$ definida por

$$
\left\{V_{0}=\{0\}, V_{1}=\{\text { eixo }-t\} \backslash\{0\}, V_{2}=V_{\text {reg }}\right\}
$$

Consideremos a forma $\ell(x, y, t)=t$. Do Teorema 2.4.2, temos

$$
\begin{aligned}
E u_{X}(0) & =\chi\left(V_{0} \cap \mathbb{B}_{\varepsilon} \cap \ell^{-1}\left(t_{0}\right)\right) \cdot E u_{X}\left(V_{0}\right) \\
& +\chi\left(V_{1} \cap \mathbb{B}_{\varepsilon} \cap \ell^{-1}\left(t_{0}\right)\right) \cdot E u_{X}\left(V_{1}\right) \\
& +\chi\left(V_{2} \cap \mathbb{B}_{\varepsilon} \cap \ell^{-1}\left(t_{0}\right)\right) \cdot E u_{X}\left(V_{2}\right) .
\end{aligned}
$$




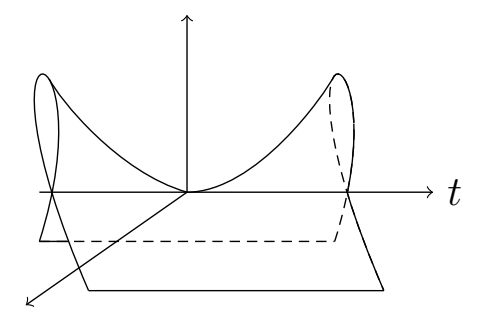

Mas, como $V_{0} \cap \mathbb{B}_{\varepsilon} \cap \ell^{-1}\left(t_{0}\right)=\emptyset$ temos então $\chi\left(V_{0} \cap \mathbb{B}_{\varepsilon} \cap \ell^{-1}\left(t_{0}\right)\right)=0$.

Por outro lado $V_{1} \cap \mathbb{B}_{\varepsilon} \cap \ell^{-1}\left(t_{0}\right)=\left\{\left(0,0, t_{0}\right)\right\}$, portanto, $\chi\left(V_{1} \cap \mathbb{B}_{\varepsilon} \cap \ell^{-1}\left(t_{0}\right)\right)=1$.

Temos $V_{2} \cap \mathbb{B}_{\varepsilon} \cap \ell^{-1}\left(t_{0}\right)=\left\{(x, y, t) / y^{2}-x^{3}-t_{0}^{2} x^{2}=0\right\} \backslash\left\{\left(0,0, t_{0}\right)\right\}$.

Com a ajuda do Teorema 2 de [11], o qual apresenta uma fórmula para a característica de Euler de uma curva plana, podemos calcular $\chi\left(V_{2} \cap \mathbb{B}_{\varepsilon} \cap \ell^{-1}\left(t_{0}\right)\right)=-1$

Pelo primeiro item da Observação 2.3.6 temos $E u_{X}\left(V_{2}\right)=E u_{X}\left(V_{\text {reg }}\right)=1$. Para calcular $E u_{X}\left(V_{1}\right)$ tomemos $t_{0} \in V_{1}$, note que em uma pequena vizinhança de $t_{0}$ temos que $V_{1}$ é difeomorfa ao produto de um pequeno disco contendo $t_{0}$ e contido no eixo $-t$ com a curva $X \cap \ell^{-1}\left(t_{0}\right)$, usando o segundo e o último item da Observação 2.3.6, obtemos $E u_{X}\left(V_{1}\right)=2$. Então:

$$
E u_{X}(0)=0 \cdot E u_{X}(0)+1.2+(-1) \cdot 1=1 .
$$

Portanto, qualquer seção não nula $\tilde{v}$ de $\tilde{T}$ sobre $\nu^{-1}\left(X \cap \partial \mathbb{B}_{\varepsilon}\right)$, levantamento de um campo de vetores radial $v$ em $0 \in X$ sobre o conjunto $X \cap \partial \mathbb{B}_{\varepsilon}$, não pode ser estendido sobre $\nu^{-1}\left(X \cap \mathbb{B}_{\varepsilon}\right)$ sem singularidade.

\subsection{Obstrução de Euler de $f$}

A obstrução de Euler de uma função $f$ foi introduzida por J.-P. Brasselet, D. Massey, A. J. Parameswaran e J. Seade em [1]. O objetivo dos autores em [1] é entender o que impede a obstrução de Euler de satisfazer a condição local de Euler para funções analíticas com singularidade isolada na origem. O "defeito" para que a condição seja satisfeita nesse caso, é chamado obstrução de Euler de $f$. 
Um invariante importante de um germe de função analítica $f:\left(\mathbb{C}^{n}, 0\right) \rightarrow(\mathbb{C}, 0)$ com ponto crítico isolado na origem é o seu número de Milnor $\mu(f)$, definido na seção 1.5 .

Este invariante fornece várias informações sobre a geometria de $f$, por exemplo, no caso em que $f$ tem ponto crítico isolado na origem os seguintes invariantes coincidem a menos de sinal.

(a) O número de Milnor de $f$ em 0 , denotado por $\mu(f)$;

(b) O número de pontos de Morse de uma Morsificação de $f$;

(c) O índice de Poincaré-Hopf do campo gradiente de $f$ conjugado $\overline{\nabla f}$.

Suponhamos agora que $(X, 0)$ seja um germe de um espaço analítico complexo mergulhado em $\mathbb{C}^{n}$. Uma das possíveis generalizações de $(c)$ é a obstrução de Euler de $f$ na origem, denotada por $E u_{f, X}(0)$.

Para o desenvolvimento deste capítulo introduziremos noções básicas sobre funções complexas definidas em espaços singulares, mas especificamente variedades analíticas. Em [24], Lê D. T. introduziu a noção de funções analíticas com singularidade isolada sobre um espaço analítico complexo $X$ com uma estratificação de Whitney $\left\{V_{i}\right\}$.

$\operatorname{Sejam~}(X, 0)$ germe de espaço analítico complexo, equidimensional e $X \subset U$ um representante do germe, com $U$ aberto de $\mathbb{C}^{n}$. Consideremos $\left\{V_{i}\right\}$ uma estratificação de Whitney de $U$, adaptada à $X$. Suponhamos que a origem seja um estrato e que o representante $\mathrm{X}$ é suficientemente pequeno para que $\{0\}$ esteja no fecho de todos os estratos. Denotaremos por $V_{i}(x)$ o estrato que contém $x \in X$.

Seja agora $f: X \rightarrow \mathbb{C}$, função analítica a qual é a restrição de uma função analítica $\tilde{f}: U \rightarrow \mathbb{C}$.

Definição 2.5.1. Um ponto $x \in X$ é um ponto singular de $f$ se $d \tilde{f}(x)\left(T_{x}\left(V_{i}(x)\right)\right)=0$, ou seja, $T_{x}\left(V_{i}(x)\right) \subset \operatorname{ker}(d \tilde{f})$.

Dizemos que $f$ tem uma singularidade isolada em $0 \in X$ relativa à estratificação de Whitney de $X$ se em uma vizinhança de 0 em $X$, a origem é o único ponto singular.

Com a construção a seguir, associaremos a $f$ um campo estratificado denotado por $\bar{\nabla}_{X} f$. Denotemos por $\bar{\nabla} \tilde{f}(x)$ o campo vetorial gradiente conjugado de $\tilde{f}$ sobre um ponto 
$x \in U$, definido por

$$
\bar{\nabla} \tilde{f}(x)=\left(\frac{\overline{\partial \tilde{f}}}{\partial x_{1}}, \cdots, \frac{\overline{\partial \tilde{f}}}{\partial x_{n}}\right),
$$

onde a barra significa a conjugação complexa.

Consideremos $f$ com uma singularidade isolada em $0 \in X$, isto implica que o núcleo $\operatorname{ker}(d \tilde{f})$ é transversal à $T_{x}\left(V_{i}(x)\right)$ para todo $x \in X \backslash\{0\}$, assim

$$
A n g\left\langle\bar{\nabla} \tilde{f}(x), T_{x}\left(V_{i}(x)\right)\right\rangle<\pi / 2
$$

onde $A n g\langle\cdot, \cdot\rangle$ denota o ângulo entre um vetor e um espaço vetorial. Então a projeção de $\bar{\nabla} \tilde{f}(x)$ sobre $T_{x}\left(V_{i}(x)\right)$, que denotaremos por $\widehat{\zeta}_{i}(x)$, não é nula.

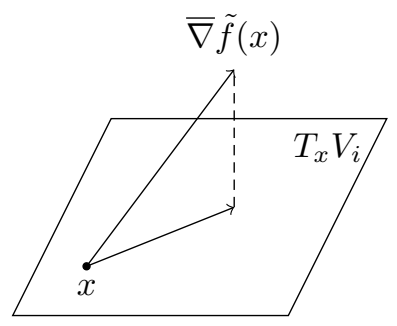

Seja $V_{j}$ um estrato tal que $V_{i} \subset \bar{V}_{j}$, e seja $\pi: U_{i} \rightarrow V_{i}$ uma vizinhança tubular de $V_{i}$ em $U$. Seguindo a construção de M.-H. Schwartz [22], temos que a condição (a) de Whitney implica que para todo ponto $y \in V_{j} \cap U_{i}$, o ângulo entre $\widehat{\zeta}_{j}(y)$ e a extensão paralela de $\widehat{\zeta}_{i}(\pi(y))$ é pequeno. Esta propriedade implica que estes dois campos de vetores são homotópicos sobre o bordo de $U_{i}$, para $U_{i}$ suficientemente pequeno. Podemos então colar os campos de vetores $\widehat{\zeta}_{i}(x)$ para obter um campo de vetores contínuo estratificado sobre $X$, que denotaremos por $\bar{\nabla}_{X} f$. Este campo de vetores é homotópico à $\left.\bar{\nabla} \tilde{f}\right|_{X}$ e temos $\bar{\nabla}_{X} f \neq 0$ para todo $x \in X \backslash\{0\}$.

Analogamente ao caso de um campo radial, utilizando a transformada de Nash definida na seção 2.2 podemos também levantar o campo $\bar{\nabla}_{X} f$ sobre $\nu^{-1}\left(X \cap \partial \mathbb{B}_{\varepsilon}\right)$ sem singularidades. Denotemos este campo por $\tilde{\bar{\nabla}}_{X} f$. O campo $\tilde{\bar{\nabla}}_{X} f$ define um cociclo de obstrução $\mathcal{O}\left(\tilde{\nabla}_{X} f\right)$, que mede a obstrução para estender $\tilde{\nabla}_{X} f$ como seção não-nula de $\widetilde{T}$ sobre $\nu^{-1}\left(X \cap \mathbb{B}_{\varepsilon}\right)$ :

$$
\mathcal{O}\left(\tilde{\bar{\nabla}}_{X} f\right) \in Z^{2 d}\left(\nu^{-1}\left(X \cap \mathbb{B}_{\varepsilon}\right), \nu^{-1}\left(X \cap \partial \mathbb{B}_{\varepsilon}\right)\right) .
$$


Desta forma temos a seguinte definição.

Definição 2.5.2. A obstrução de Euler de $f$ na origem, denotada por $E u_{f, X}(0)$, é o inteiro obtido pela avaliação do cociclo de obstrução $\mathcal{O}\left(\tilde{\nabla}_{X} f\right)$ sobre a classe fundamental $\left(\nu^{-1}\left(X \cap \mathbb{B}_{\varepsilon}\right), \nu^{-1}\left(X \cap \partial \mathbb{B}_{\varepsilon}\right)\right)$, ou seja,

$$
E u_{f, X}(0):=\mathcal{O}\left(\tilde{\bar{\nabla}}_{X} f\right) \cap\left(\nu^{-1}\left(X \cap \mathbb{B}_{\varepsilon}\right), \nu^{-1}\left(X \cap \partial \mathbb{B}_{\varepsilon}\right)\right)
$$

Note que todas essas definições e construções também valem quando consideramos $f$ como a restrição de uma função analítica real. Podemos tomar $f$ como a função distância a 0 em $X$, então $\bar{\nabla}_{X} f$ é um campo de vetores radial e o invariante $E u_{f, X}(0)$ é a obstrução de Euler de $X$ na origem.

Observamos que a métrica Hermitiana usual em $\mathbb{C}^{n}$ define uma métrica Riemanniana, a qual permite identificar o fibrado vetorial $T \mathbb{C}^{n}$ com o fibrado cotangente holomorfo $T^{*} \mathbb{C}^{n}$. Com essa identificação, $d \tilde{f}$ corresponde ao campo de vetores gradiente conjugado.

A razão para se considerar o campo de vetores gradiente conjugado $\bar{\nabla}_{X} f$ e não o campo gradiente usual $\nabla_{X} f$ é dada pelo seguinte lema.

Lema 2.5.3. O campo vetorial $\bar{\nabla}_{X} f$ é o levantamento, a menos de homotopia, de um campo de vetores constante em $\mathbb{C}$, via $d \tilde{f}$.

Demonstração. Primeiramente, observamos que $\bar{\nabla}_{X} f$ é homotópico à $\left.\bar{\nabla} \tilde{f}\right|_{X}$ e o vetor gradiente satisfaz,

$$
d \tilde{f}(\bar{\nabla} \tilde{f}(x))=\|\bar{\nabla} \tilde{f}(x)\|^{2} \in \mathbb{R}_{+}^{*}, \forall x \in X \backslash\{0\}
$$

Isto significa que ele é um levantamento, a menos de uma homotopia, de um campo vetorial constante em um pequeno disco $\mathbb{D}_{\eta} \subset \mathbb{C}$.

Neste trabalho, consideraremos somente funções analíticas complexas.

Observação 2.5.4. Se 0 é um ponto suave de $X$ e também um ponto regular de $f$, então $E u_{f, X}(0)=0$. Na Proposição 2.5.6. provaremos esse resultado em uma situação mais geral. 
Definição 2.5.5. Seja $(X, 0) \subset(U, 0)$ um germe de variedade analítica em $\mathbb{C}^{n}$, com uma estratificação de Whitney $\left\{V_{i}\right\}$ e seja $f:(X, 0) \rightarrow(\mathbb{C}, 0)$ um germe de aplicação analítica que vem de uma restrição de um germe de aplicação analítica regular $\tilde{f}:(U, 0) \rightarrow(\mathbb{C}, 0)$. Dizemos que 0 é um ponto geral de $f($ ou que $f:(X, 0) \rightarrow(\mathbb{C}, 0)$ é geral em 0) se o hiperplano ker $(d \widetilde{f}(0))$ é transversal em $\mathbb{C}^{n}$ a todo limite de espaços tangentes $T_{x_{n}}\left(V_{i}\right)$, para todo $V_{i}$ e toda sequência $\left\{x_{n}\right\} \in V_{i}$ convergindo para 0 .

Observemos que para toda $f$ como acima, o conjunto de pontos gerais é não vazio e aberto em cada estrato (aberto) de $X$, essencialmente pelo Teorema de Sard 1.1.15).

A definição anterior é equivalente a dizer que, com uma mudança de coordenadas apropriada, $\widetilde{f}$ é uma forma linear em $U$ e é genérica com respeito à $X$.

Proposição 2.5.6. Seja 0 um ponto geral de $f:(X, 0) \rightarrow(\mathbb{C}, 0)$. Então,

$$
E u_{f, X}(0)=0
$$

Demonstração. Como primeiro passo definimos a aplicação

$$
\widetilde{T} \subset(U \times G(d, n)) \times \mathbb{C}^{n} \stackrel{\widetilde{F}}{\rightarrow} \mathbb{D}_{\eta} \subset \mathbb{C}
$$

por $\widetilde{F}(x, P, y)=d \widetilde{f}_{x}(y)$. Como 0 é um ponto geral de $f$, então $\widetilde{K}=\widetilde{T} \cap \widetilde{F}^{-1}(0)$ é um subfibrado de $\widetilde{T}$ de codimensão (complexa) 1 e $d \widetilde{F}$ leva o complemento ortogonal de $\widetilde{K}$ isomorficamente sobre $T \mathbb{D}_{\eta}$.

Agora, toda $\widetilde{f}$ que define uma singularidade isolada em 0 em $X$, determina um subfibrado $Q$ de $\left.T \mathbb{C}^{n}\right|_{X \backslash\{0\}}$ sempre transversal à $k e r(d \widetilde{f})$, e a restrição de $d \widetilde{f}$ à $Q$ é um isomorfismo entre $Q$ e $T \mathbb{D}_{\eta}$.

Isto implica que cada vetor não-nulo em $\mathbb{D}_{\eta}$ se levanta de forma compatível, a um campo de vetores em $X \backslash\{0\}$ e também como uma seção de $\left.\widetilde{T}\right|_{X}$. O passo final é apenas notar que como no Lema 2.5.3, o vetor gradiente $\bar{\nabla}_{X} f$ pode ser obtido por um levantamento de tais campos vetoriais.

A proposição que apresentaremos a seguir, cuja demonstração encontra-se em [17], será utilizada em resultados do capítulo seguinte. 
Proposição 2.5.7. Seja $f:(X, 0) \rightarrow(\mathbb{C}, 0)$ analítica em 0 . Então existe um aberto de Zariski $\Omega_{f}$ dentro do espaço das formas lineares em $\mathbb{C}^{n}$, tal que para todo $\ell \in \Omega_{f}$, 0 é um ponto geral para a aplicação $f+\lambda \ell: X \rightarrow \mathbb{C}$, para todo $\lambda \in \mathbb{C}^{*}$ suficientemente pequeno. 



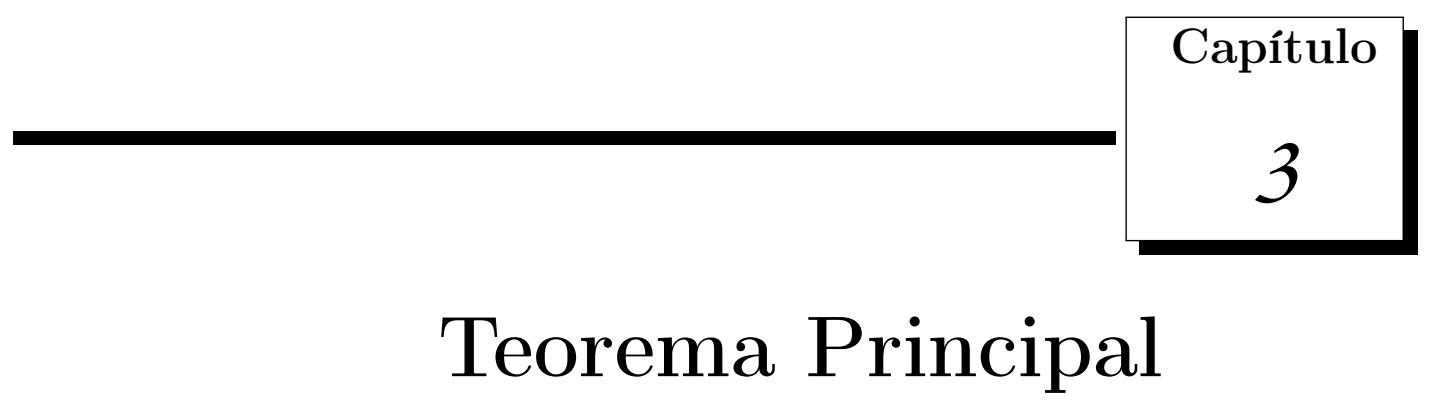

Neste capítulo, apresentaremos o resultado mais importante deste trabalho. O qual apresenta uma comparação entre a obstrução de Euler e a obstrução de Euler de uma função.

\subsection{Teorema Principal}

Teorema 3.1.1. Seja $f:(X, 0) \rightarrow(\mathbb{C}, 0)$ com singularidade isolada na origem $e\left\{V_{i}\right\}$ uma estratificação de Whitney de X. Então,

$$
E u_{X}(0)=\left(\sum_{i} \chi\left(V_{i} \cap \mathbb{B}_{\varepsilon} \cap f^{-1}\left(t_{0}\right)\right) \cdot E u_{X}\left(V_{i}\right)\right)+E u_{f, X}(0)
$$

onde $\varepsilon$ é suficientemente pequeno, $t_{0} \in \mathbb{C} \backslash\{0\}$ é tal que $\left\|t_{0}\right\|<<1$ e E $u_{X}\left(V_{i}\right)$ é a obstrução de Euler de $X$ em qualquer ponto do estrato $V_{i}$.

Note que este resultado generaliza o Teorema 2.4.2, basta considerar $f$ uma forma linear genérica e utilizar a Proposição 2.5.6.

A demonstração deste resultado segue do Lema 3.1.2. Primeiramente, fixaremos algumas notações. Seja $\varepsilon>0$ suficientemente pequeno tal que toda esfera $\mathbb{S}_{\gamma}$ em $U$ centrada em 0 e com raio $\gamma \leq \varepsilon$ intersecta transversalmente todo estrato em $X \backslash\{0\}$, o qual existe pelo Teorema de Bertini-Sard 2.3.4. Para cada $t \in \mathbb{C}$ denotaremos por $Y_{t}:=f^{-1}(t)$. Seja 
$\eta>0$ pequeno o suficiente tal que para cada $t$ em $\mathbb{D}_{\eta}$ de raio $\eta$ e centro em $0 \in \mathbb{C}$, a hipersuperfície $Y_{t}$ intersecta transversalmente a esfera $\mathbb{S}_{\varepsilon}$. Agora, tome $\varepsilon^{\prime} \operatorname{com} 0<\varepsilon^{\prime}<\varepsilon$, e um ponto $t_{0} \in \mathbb{D}_{\eta}$ tal que $Y_{t_{0}}$ não encontra a esfera $\mathbb{S}_{\varepsilon^{\prime}}$. Note que o estrato $V_{i}$ intersecta $Y_{t_{0}}:=f^{-1}\left(t_{0}\right)$ transversalmente e induz uma estratificação de Whitney para este espaço.

Lema 3.1.2. Seja $(X, 0)$ um germe de variedade analitica complexa, equidimensional de dimensão complexa d. Seja $f:(X, 0) \rightarrow(\mathbb{C}, 0)$ analítica. Existe um campo vetorial estratificado w sobre $X_{\varepsilon^{\prime}, \varepsilon}=X \cap\left(\mathbb{B}_{\varepsilon} \backslash \operatorname{Int}\left(\mathbb{B}_{\varepsilon^{\prime}}\right)\right)$ onde $0<\varepsilon^{\prime}<\varepsilon$ tal que:

1. $w$ coincide com $\bar{\nabla}_{X} f$ sobre $X \cap \partial \mathbb{B}_{\varepsilon^{\prime}}$ e w é radial sobre $X \cap \partial \mathbb{B}_{\varepsilon}$;

2. $w$ é tangente $\grave{a} Y_{t_{0}}$;

3. $w$ tem apenas um número finito de zeros, e todos estão contidos em $Y_{t_{0}}$;

4. em cada zero a de w o campo w é transversalmente radial ao estrato que contém a (ou seja, o campo w é transversal à fronteira de uma vizinhança tubular do estrato).

Para a demonstração do Teorema 3.1.1, também usaremos o seguinte resultado.

Teorema 3.1.3 (Théorème de proporcionalité, [2]). Seja v um campo de vetores estratificado em um espaço analítico complexo equidimensional $X$ mergulhado em $\mathbb{C}^{n}$ com um zero isolado em a e seja $I(v, a)$ o indice de Schwartz de v em a. Seja $\varepsilon>0$ suficientemente pequeno (dado pelo Teorema de Bertini-Sard 2.3.4). A restrição de v à interseção $X \cap \mathbb{S}_{\varepsilon}(a)$ pode ser levantada como uma seção não-nula $\tilde{v}$ do fibrado de Nash $\tilde{T}$ de X. Seja $\mathcal{O}(v, a)$ a obstrução para estender $\tilde{v}$ como uma seção não-nula de $\tilde{T}$ sobre $\nu^{-1}\left(X \cap \mathbb{B}_{\varepsilon}(a)\right)$. Então,

$$
\mathcal{O}(v, a)=I(v, a) \cdot E u_{X}(a)
$$

Observação 3.1.4. O índice de Schwartz é definido para campos de vetores definidos em variedades singulares, para mais detalhes sugerimos [2] e [3]. Para a prova do teorema principal deste trabalho, construíremos um campo estratificado $w$ sobre $X$ com singularidades isoladas, de tal forma que em cada singularidade $a$, o campo é transversalmente radial ao estrato contendo $a$. Neste caso, o índice de Schwartz $I(w ; a)$ de $w$ em $a$ coincide o índice de Poincaré-Hopf $\operatorname{Ind}_{P H}(w, a)$ (definido na seção 1.1) de $w$ em $a$ no estrato que 
contém $a$. Temos também que, se a é um ponto regular de X, então o índice de Schwartz é o índice de Poincaré-Hopf.

Primeiramente, vamos demonstrar o Teorema 3.1.1 assumindo o Lema 3.1 .2 e faremos a demonstração do Lema 3.1 .2 na seção 3.3 .

Demonstração do Teorema 3.1.1: Se $\xi$ é um campo de vetores estratificado em uma vizinhança da origem em $X$ transversal à uma pequena esfera $\mathbb{S}_{\varepsilon}$, apontando para fora, então $\xi$ é homotópico à um campo de vetores radial. Portanto, pela teoria de obstrução, para calcular a obstrução de Euler é suficiente considerar campos de vetores transversais à $\mathbb{S}_{\varepsilon}$.

A restrição do campo de vetores $w$, do Lema 3.1 .2 sobre $\partial\left(X_{\varepsilon^{\prime}, \varepsilon}\right)$ é um campo de vetores estratificado, então pode ser levantado como uma seção $\tilde{w}$ do fibrado de Nash $\tilde{T}$ sobre $\nu^{-1}\left(\partial\left(X_{\varepsilon^{\prime}, \varepsilon}\right)\right)$ pelo Lema 2.3.3. Denotaremos por $\mathcal{O}\left(\tilde{w}, \nu^{-1}\left(X_{\varepsilon}\right)\right)$ a obstrução em se estender $\tilde{w}$ sobre $\nu^{-1}\left(X_{\varepsilon}\right)$. Temos,

$$
\mathcal{O}\left(\tilde{w}, \nu^{-1}\left(X_{\varepsilon}\right)\right)=\mathcal{O}\left(\tilde{w}, \nu^{-1}\left(X_{\varepsilon^{\prime}}\right)\right)+\mathcal{O}\left(\tilde{w}, \nu^{-1}\left(X_{\varepsilon^{\prime}, \varepsilon}\right)\right)
$$

Segue do Lema $3.1 .2(1)$,

$$
E u_{X}(0)=E u_{f, X}(0)+\mathcal{O}\left(\tilde{w}, \nu^{-1}\left(X_{\varepsilon^{\prime}, \varepsilon}\right)\right)
$$

Pelo item (3) do Lema 3.1.2, a contribuição de $\mathcal{O}\left(\tilde{w}, \nu^{-1}\left(X_{\varepsilon^{\prime}, \varepsilon}\right)\right)$ está concentrada em $\nu^{-1}\left(Y_{t_{0}} \cap \mathbb{B}_{\varepsilon}\right)$. Os itens (3) e (4) do Lema 3.1.2, juntamente com o Teorema 3.1.3 e a observação 3.1 .4 implicam que a contribuição de cada singularidade $a$ de $w$ para $\mathcal{O}\left(\tilde{w}, \nu^{-1}\left(X_{\varepsilon^{\prime}, \varepsilon}\right)\right)$ é $E u_{X}(a)$-vezes o índice local de Poincaré-Hopf de $w$ em $a$, visto como um campo de vetores no estrato $V_{i}(a)$. Logo,

$$
\mathcal{O}\left(\tilde{w}, \nu^{-1}\left(X_{\varepsilon^{\prime}, \varepsilon}\right)\right)=\sum_{a} E u_{X}(a) \cdot \operatorname{Ind}_{P H}(w, a)
$$

Pelos itens (2) e (4) do Lema 3.1.2 e pelo Teorema de Poincaré-Hopf (1.1.41) a soma dos índices de Poincaré-Hopf da restrição de $w$ à $V_{i} \cap Y_{t_{0}}$ é $\chi\left(V_{i} \cap Y_{t_{0}} \cap \mathbb{B}_{\varepsilon}\right)$.

Portanto, 


$$
\begin{gathered}
\mathcal{O}\left(\tilde{w}, \nu^{-1}\left(X_{\varepsilon^{\prime}, \varepsilon}\right)\right)=\sum_{i}\left(\sum_{a \in V_{i}} \operatorname{Ind}_{P H}(w, a)\right) E u_{X}\left(V_{i}\right) \\
=\sum_{i} \chi\left(V_{i} \cap \mathbb{B}_{\varepsilon} \cap f^{-1}\left(t_{0}\right)\right) \cdot E u_{X}\left(V_{i}\right) .
\end{gathered}
$$

Corolário 3.1.5. Seja $f:(X, 0) \rightarrow(\mathbb{C}, 0)$ germe com singularidade isolada na origem. Seja l uma forma linear em $\Omega_{f}$ como na Proposição 2.5.7, e seja $\lambda \in \mathbb{C}^{*}$ tal que $f_{\lambda}:=$ $f+\lambda \ell$ seja geral na origem. Sejam $F_{f}$ e $F_{f_{\lambda}}$ as fibras de Milnor de $f$ e $f_{\lambda}$, respectivamente, em $X$. Então,

$$
E u_{f, X}(0)=\sum_{i}\left(\chi\left(V_{i} \cap F_{f_{\lambda}}\right)-\chi\left(V_{i} \cap F_{f}\right)\right) \cdot E u_{X}\left(V_{i}\right) .
$$

Demonstração. Do Teorema 3.1.1. temos

$$
E u_{f, X}(0)=E u_{X}(0)-\left(\sum_{i} \chi\left(V_{i} \cap \mathbb{B}_{\varepsilon} \cap f^{-1}\left(t_{0}\right)\right) \cdot E u_{X}\left(V_{i}\right)\right) .
$$

Segue dos Teoremas 3.1.1 1.5.5,

$E u_{f, X}(0)=\left(\sum_{i} \chi\left(V_{i} \cap \mathbb{B}_{\varepsilon} \cap f_{\lambda}^{-1}\left(t_{0}\right)\right) \cdot E u_{X}\left(V_{i}\right)\right)+E u_{f_{\lambda}, X}(0)-\left(\sum_{i} \chi\left(V_{i} \cap F_{f}\right) \cdot E u_{X}\left(V_{i}\right)\right)$.

Da Proposição 2.5.6 segue $E u_{f_{\lambda}, X}(0)=0$, portanto,

$$
E u_{f, X}(0)=\sum_{i}\left(\chi\left(V_{i} \cap F_{f_{\lambda}}\right)-\sum_{i} \chi\left(V_{i} \cap F_{f}\right)\right) \cdot E u_{X}\left(V_{i}\right) .
$$

Observação 3.1.6. Se $X=\mathbb{C}^{d}$ e $f:\left(\mathbb{C}^{d}, 0\right) \rightarrow(\mathbb{C}, 0)$ é uma função analítica com singularidade isolada na origem e número de Milnor $\mu(f)$, então

$$
E u_{f, \mathbb{C}^{d}}(0)=(-1)^{d} \mu(f)
$$


Pois, pelo Teorema 3.1.1.

$$
E u_{f, \mathbb{C}^{d}}(0)=E u_{\mathbb{C}^{d}}(0)-\chi\left(\mathbb{C}^{d} \cap \mathbb{B}_{\varepsilon} \cap f^{-1}\left(t_{0}\right)\right) \cdot E u_{X}\left(\mathbb{C}^{d}\right)
$$

Logo,

$$
E u_{f, \mathbb{C}^{d}}(0)=E u_{\mathbb{C}^{d}}(0)-\chi\left(F_{f}\right) \cdot E u_{\mathbb{C}^{d}}(0)
$$

Do primeiro item da Observação 2.3.6, temos $E u_{\mathbb{C}^{d}}(0)=1$ e a característica de EulerPoincaré da fibra de Milnor de $f$ é igual à $1+(-1)^{d-1} \mu(f)$ (ver [18]). Assim,

$$
E u_{f, \mathbb{C}^{d}}(0)=1-1-(-1)^{d-1} \mu(f)
$$

Portanto,

$$
E u_{f, \mathbb{C}^{d}}(0)=(-1)^{d} \mu(f)
$$

Observação 3.1.7. Sejam $f:\left(\mathbb{C}^{d}, 0\right) \rightarrow(\mathbb{C} ; 0)$ uma função analítica com singularidade isolada na origem, $\ell: \mathbb{C}^{d} \rightarrow \mathbb{C}$ uma forma linear genérica e $X=f^{-1}(0)$, pelo Teorema 2.4 .2 temos,

$$
E u_{X}(0)=\chi\left(\{0\} \cap B_{\varepsilon} \cap \ell^{-1}\left(t_{0}\right)\right) \cdot E u_{X}(0)+\chi\left(X \backslash\{0\} \cap B_{\varepsilon} \cap \ell^{-1}\left(t_{0}\right)\right) \cdot E u_{X}(X \backslash\{0\}) .
$$

Temos, $\left(\{0\} \cap B_{\varepsilon} \cap \ell^{-1}\left(t_{0}\right)\right)=\emptyset, \log 0 \chi\left(\{0\} \cap B_{\varepsilon} \cap \ell^{-1}\left(t_{0}\right)\right)=0$, temos também, $E u_{X}(X \backslash\{0\})=1$ e como $\ell^{-1}\left(t_{0}\right)$ não contém a origem, temos $X \backslash\{0\} \cap B_{\varepsilon} \cap \ell^{-1}\left(t_{0}\right)=$ $X \cap B_{\varepsilon} \cap \ell^{-1}\left(t_{0}\right)$. Assim,

$$
E u_{X}(0)=\chi\left(f^{-1}(0) \cap B_{\varepsilon} \cap \ell^{-1}\left(t_{0}\right)\right) .
$$

Considerando $g=(f, \ell)$ e a ICSI definida por $g^{-1}\left(0, t_{0}\right)$ segue, da igualdade acima, que a obstrução de Euler em $X=f^{-1}(0)$ é a característica de Euler da fibra de Milnor da ICSI $g^{-1}\left(0, t_{0}\right)$, ou seja, 


$$
E u_{f^{-1}(0)}(0)=\chi\left(F_{(f, \ell)}\right)
$$

Logo, a obstrução de Euler, neste caso, é dada em função do número de Milnor de $g=(f, \ell)$,

$$
E u_{f^{-1}(0)}(0)=1+(-1)^{d-2} \mu((f, \ell)) .
$$

Com essa observação e com a observação 3.1.6, podemos perceber que $E u_{f, \mathbb{C}^{d}}(0)$ é, a menos de sinal, o número de Milnor de $f$, porém $E u_{f^{-1}(0)}(0)$ se relaciona com a característica de Euler de uma seção de $f^{-1}(0)$, ou seja, $E u_{f^{-1}(0)}(0)=\chi\left(F_{(f, \ell)}\right)$.

Observação 3.1.8. Se $X=\mathbb{C}^{d}$ e $f:\left(\mathbb{C}^{d}, 0\right) \rightarrow(\mathbb{C}, 0)$ é uma função analítica com singularidade isolada na origem, pela observação anterior,

$$
E u_{f, \mathbb{C}^{d}}(0)=(-1)^{d} \mu(f)
$$

Então, $\mu(f)=\operatorname{deg}(\nabla f)$.

De fato, como $X=\mathbb{C}^{d}$, temos $\tilde{X}=\mathbb{C}^{d}$ e $\tilde{T}=T \mathbb{C}^{d}$. Assim,

$$
\begin{gathered}
E u_{f, \mathbb{C}^{d}}(0)=\mathcal{O}\left(\bar{\nabla} f, \nu^{-1}\left(X_{\varepsilon}\right)\right)=E u_{\mathbb{C}^{d}}(0) \cdot \operatorname{Ind}_{P H}(\bar{\nabla} f, 0) \\
=\operatorname{Ind}_{P H}(\bar{\nabla} f, 0)=(-1)^{d} \operatorname{Ind}_{P H}(\nabla f, 0)=(-1)^{d} \operatorname{deg}(\nabla f) .
\end{gathered}
$$

Portanto,

$$
\mu(f)=\operatorname{deg}(\nabla f)
$$

Exemplo 3.1.9. Seja $f: \mathbb{C}^{3} \rightarrow \mathbb{C}$ dada por $f(x, y, z)=x^{3}+y^{4}+z^{5}$.

Do Teorema 1.5 .8 ,

$$
\mu(f)=\operatorname{dim}_{\mathbb{C}} \frac{\mathcal{O}_{n}}{J(f)} .
$$

Logo, 


$$
\mu(f)=\operatorname{dim}_{\mathbb{C}} \frac{\mathbb{C}\{x, y, z\}}{\left\langle x^{2}, y^{3}, z^{4}\right\rangle}=24
$$

Da observação 3.1 .6 segue que,

$$
E u_{f, \mathbb{C}^{3}}(0)=(-1)^{3} \mu(f)=-24
$$

\subsection{Lemas}

O campo de vetores $w$ do Lema 3.1.2 é definido como a soma de dois campos de vetores que construiremos nos Lemas 3.2.1 e 3.2.2.

Para os próximos resultados, fixaremos algumas notações. Seja $\eta^{\prime}>0$ suficientemente pequeno com respeito a $\eta$, tal que $\mathbb{D}_{\eta^{\prime}}\left(t_{0}\right)$ está contido no interior de $\mathbb{D}_{\eta}$ e $f^{-1}\left(\mathbb{D}_{\eta^{\prime}}\left(t_{0}\right)\right)$ não tem interseção com a esfera $\mathbb{S}_{\varepsilon^{\prime}}$.

Lema 3.2.1. Existe um campo de vetores estratificado $r$ em $\mathbb{B}_{\varepsilon} \cap f^{-1}\left(\mathbb{D}_{\eta}\right)$ satisfazendo:

1. A restrição de $r$ à $\mathbb{S}_{\varepsilon} \cap f^{-1}\left(\mathbb{D}_{\eta}\right)$ é tangente a todas as fibras $f^{-1}(t)$ e é transversal $\grave{a} \mathbb{S}_{\varepsilon}$, apontando para fora;

2. $r$ é tangente à fibra $f^{-1}\left(t_{0}\right)$, onde tem somente singularidades isoladas. Além disso, em cada zero, $r$ é transversalmente radial (em $\left.f^{-1}\left(t_{0}\right)\right)$ a cada estrato que contém o zero;

3. $r$ é tangente à fibra $f^{-1}(t)$, para todo $t \in \mathbb{D}_{\eta^{\prime}}\left(t_{0}\right)$.

Demonstração. Primeiramente construiremos $r$ em $f^{-1}\left(t_{0}\right)$ satisfazendo (2) e apontando para fora de $\mathbb{S}_{\varepsilon}$. Denotemos $f^{-1}\left(t_{0}\right) \cap \mathbb{B}_{\varepsilon}$ por $F$. As componentes conexas das interseções $V_{i} \cap F$ definem uma estratificação de Whitney de $F$. Esta define uma filtração $F_{n_{1}}, \ldots, F_{n_{r}}$ de $F$ onde $0 \leq n_{1}<\ldots<n_{r}=d-1$ e $F_{n_{i}}$ é a união dos estratos de dimensão $\leq n_{i}$. Então, $F_{n_{1}}$ é uma variedade suave e $F_{n_{r}}=F$. Construíremos $r$ por indução em $n_{i}$.

Seja $v_{1}$ um campo de vetores suave em $F_{n_{1}}$, o qual tem singularidades isoladas e é transversal à $X \cap \mathbb{S}_{\varepsilon}$ apontando para fora. Pelo Teorema de Poincaré-Hopf, o índice total de $v_{1}$ em $F_{n_{1}}$ é $\chi\left(F_{n_{1}}\right)$. 
Como em [22], podemos estender esse campo de vetores de modo transversalmente radial. Temos um campo de vetores estratificado $\bar{v}_{1}$ em um tubo fechado $T_{1}$ em $F$ em torno de $F_{n_{1}}$, o qual tem os mesmos pontos singulares de $v_{1}$ e o mesmo índice de Schwartz. Se o tubo é suficientemente pequeno, a extensão fica transversal à $X \cap \mathbb{S}_{\varepsilon}$ apontando para fora.

Agora estenderemos $\bar{v}_{1}$ para $\left(F_{n_{2}} \backslash \operatorname{Int}\left(T_{1}\right)\right) \cap \mathbb{B}_{\varepsilon}$. Este espaço é uma variedade suave com fronteira e bicos. A restrição de $\bar{v}_{1}$ à $F_{n_{2}} \cap \partial T_{1} \cap \mathbb{B}_{\varepsilon}$ é transversal à fronteira e aponta para fora. Podemos estender este campo de vetores como um campo de vetores contínuo ([7]) para a fronteira inteira com bicos, tal que ele aponta para fora. Em particular, este campo de vetores é transversal à $X \cap \mathbb{S}_{\varepsilon}$ apontando para fora. Ele se estende como um campo de vetores contínuo em $\left(F_{n_{2}} \backslash \operatorname{Int}\left(T_{1}\right)\right) \cap \mathbb{B}_{\varepsilon}$ com singularidades isoladas. Em cada componente conexa de $\left(F_{n_{2}} \backslash \operatorname{Int}\left(T_{1}\right)\right) \cap \mathbb{B}_{\varepsilon}$, a soma dos índices de Poincaré-Hopf deste campo de vetores é igual a característica de Euler da componente. Isto define um campo de vetores contínuo $v_{2}$ em $\left(F_{n_{2}} \cup T_{1}\right) \cap \mathbb{B}_{\varepsilon}$.

Como em [22], podemos estender $v_{2}$ de modo transversalmente radial. Temos um campo de vetores estratificado $\bar{v}_{2}$ entrando em uma vizinhança fechada $T_{2}$ de $F_{n_{2}} \cap \mathbb{B}_{\varepsilon}$, com os mesmos pontos singulares de $v_{2}$ e os mesmos índices de Schwartz. Além disso, escolhendo $T_{2}$ suficientemente pequeno, $\bar{v}_{2}$ é transversal à $X \cap \mathbb{S}_{\varepsilon}$ apontando para fora, por continuidade.

Agora, seja $1 \leq s<r$. Suponha que já temos o campo de vetores $v_{s}$ em $\left(F_{n_{s}} \cup T_{s-1}\right) \cap \mathbb{B}_{\varepsilon}$ e a extensão $\bar{v}_{s}$ um campo de vetores estratificado em uma vizinhança fechada $T_{s}$ de $F_{n_{s}} \cap \mathbb{B}_{\varepsilon}$, o qual é transversalmente radial, com os mesmos pontos singulares e os mesmos índices de $v_{s}$ (por convenção, $T_{-1}=\emptyset$ ). Podemos assumir que $\bar{v}_{s}$ é transversal à $X \cap \mathbb{S}_{\varepsilon}$ apontando para fora.

Prosseguimos, como acima, para obter uma extensão de $\bar{v}_{s}$ para $\left(F_{n_{s+1}} \cup T_{s}\right) \cap \mathbb{B}_{\varepsilon}$ com singularidades isoladas, transversal à $X \cap \mathbb{B}_{\varepsilon}$ apontando para fora. A soma dos índices de Poincaré-Hopf deste campo de vetores em cada componente conexa de $\left(F_{n_{s+1}} \backslash \operatorname{Int}\left(T_{s}\right)\right) \cap \mathbb{B}_{\varepsilon}$ é igual a característica de Euler desta componente.

Finalmente, obtemos um campo de vetores $r:=v_{r}$ em $F_{n_{r}}=F$ com singularidades isoladas contidas em cada $\left(F_{n_{s}} \backslash \operatorname{Int}\left(T_{s-1}\right)\right) \cap \mathbb{B}_{\varepsilon}$, para $s=1, \ldots, r$. Pela construção, o 
índice de Schwartz de $r$ em um ponto singular contido em algum $\left(F_{n_{s}} \backslash \operatorname{Int}\left(T_{s-1}\right)\right) \cap \mathbb{B}_{\varepsilon}$ é igual ao índice de Poincaré-Hopf de $v_{s}$, o qual é também a restrição de $r$.

Este campo de vetores satisfaz $(2)$ e aponta para fora de $\mathbb{S}_{\varepsilon}$.

Pelo Teorema de Fibração de Lê [Teorema 1.3 [25]] a função $f$ determina uma fibração estratificada localmente trivial de $\mathbb{B}_{\varepsilon} \cap f^{-1}\left(\mathbb{D}_{\eta}\right) \backslash f^{-1}(0)$ sobre $\mathbb{D}_{\eta} \backslash\{0\}$. Esta induz, em particular, uma fibração trivial sobre $\mathbb{D}_{\eta^{\prime}}\left(t_{0}\right)$. Portanto, $r$ pode ser estendido, como um produto, para todas as fibras sobre $\mathbb{D}_{\eta^{\prime}}\left(t_{0}\right)$, satisfazendo a condição (3).

Escolhemos $\varepsilon^{\prime \prime}, \varepsilon^{\prime}<<\varepsilon^{\prime \prime}<\varepsilon$, tal que a restrição de $f$ à $f^{-1}\left(\mathbb{D}_{\eta}\right) \cap\left(\mathbb{B}_{\varepsilon} \backslash \operatorname{Int}\left(\mathbb{B}_{\varepsilon^{\prime \prime}}\right)\right)$ é uma fibração trivial. Desde que $\mathbb{D}_{\eta}$ se retrai para $\mathbb{D}_{\eta^{\prime}}\left(t_{0}\right)$, o campo de vetores $r$ pode se estender para $S_{\varepsilon} \cap f^{-1}\left(\mathbb{D}_{\eta}\right)$ sendo tangentes as fibras de $f$ e transversal à esfera $S_{\varepsilon}$, apontando para fora.

Usando uma adequada partição da unidade, podemos estender $r$ como zero no complemento de uma vizinhança de $\left(f^{-1}\left(\mathbb{D}_{\eta}\right) \cap\left(\mathbb{B}_{\varepsilon} \backslash \operatorname{Int}\left(\mathbb{B}_{\varepsilon^{\prime \prime}}\right)\right)\right) \cup f^{-1}\left(\mathbb{D}_{\eta^{\prime}}\left(t_{0}\right)\right)$.

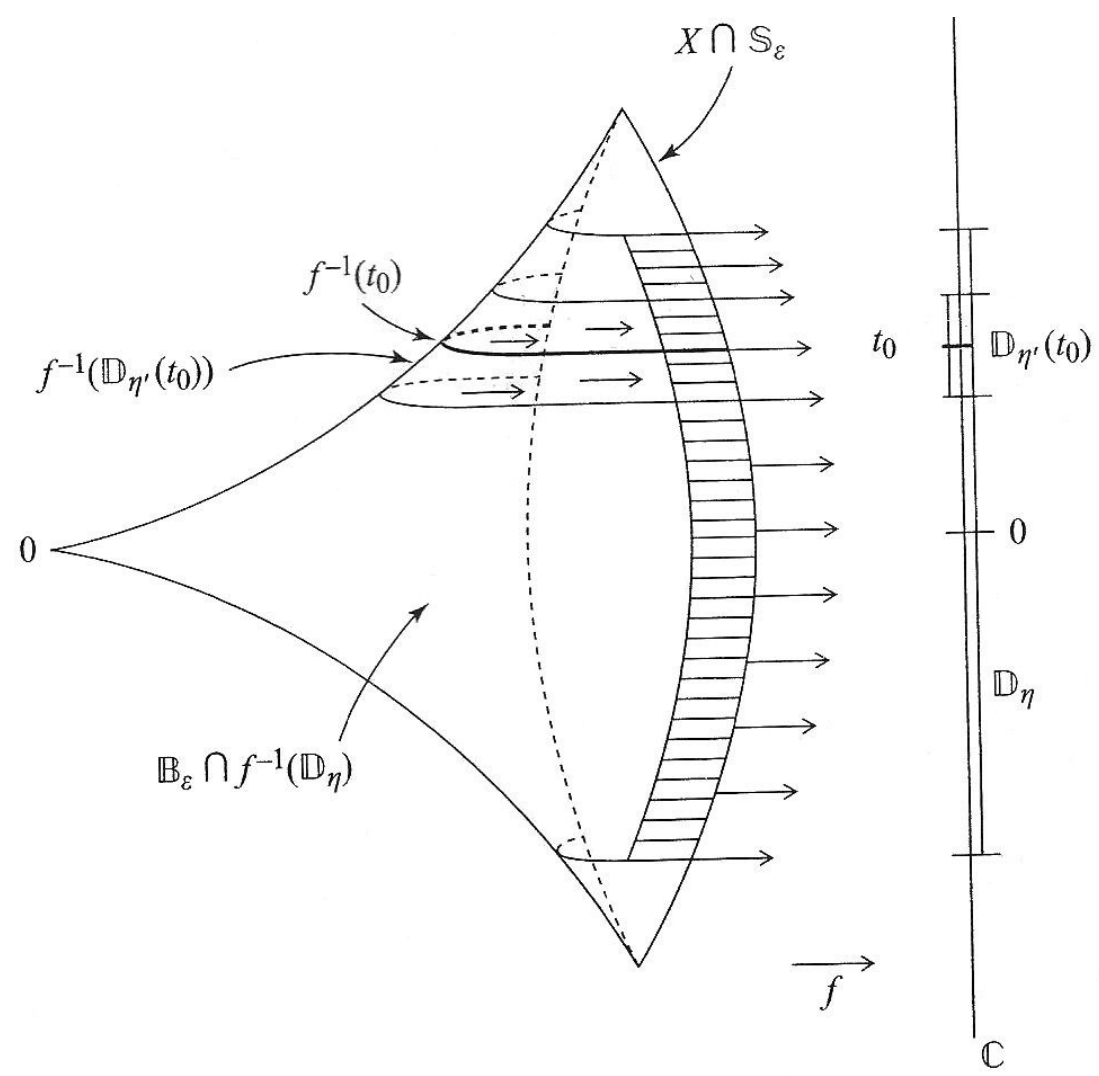


Note que as afirmações (1) e (2) do Lema 3.2.1 implicam que para cada estrato $Y_{t_{0}} \cap V_{i} \cap \mathbb{B}_{\varepsilon}$, a soma dos índices de Poincaré-Hopf da restrição de $r$ é $\chi\left(Y_{t_{0}} \cap V_{i} \cap \mathbb{B}_{\varepsilon}\right)$.

Lema 3.2.2. Existe um campo de vetores estratificado u definido em $\left(\mathbb{B}_{\varepsilon} \cap f^{-1}\left(\mathbb{D}_{\eta}\right)\right) \backslash\{0\}$ e satisfazendo

1. $u$ é tangente à $\mathbb{S}_{\varepsilon}$;

2. O conjunto de zeros de u está em $f^{-1}\left(t_{0}\right)$ e u é transversalmente radial a $f^{-1}\left(t_{0}\right)$;

3. $u$ é transversal à $X \cap f^{-1}\left(\partial \mathbb{D}_{\eta}\right)$, apontando para fora.

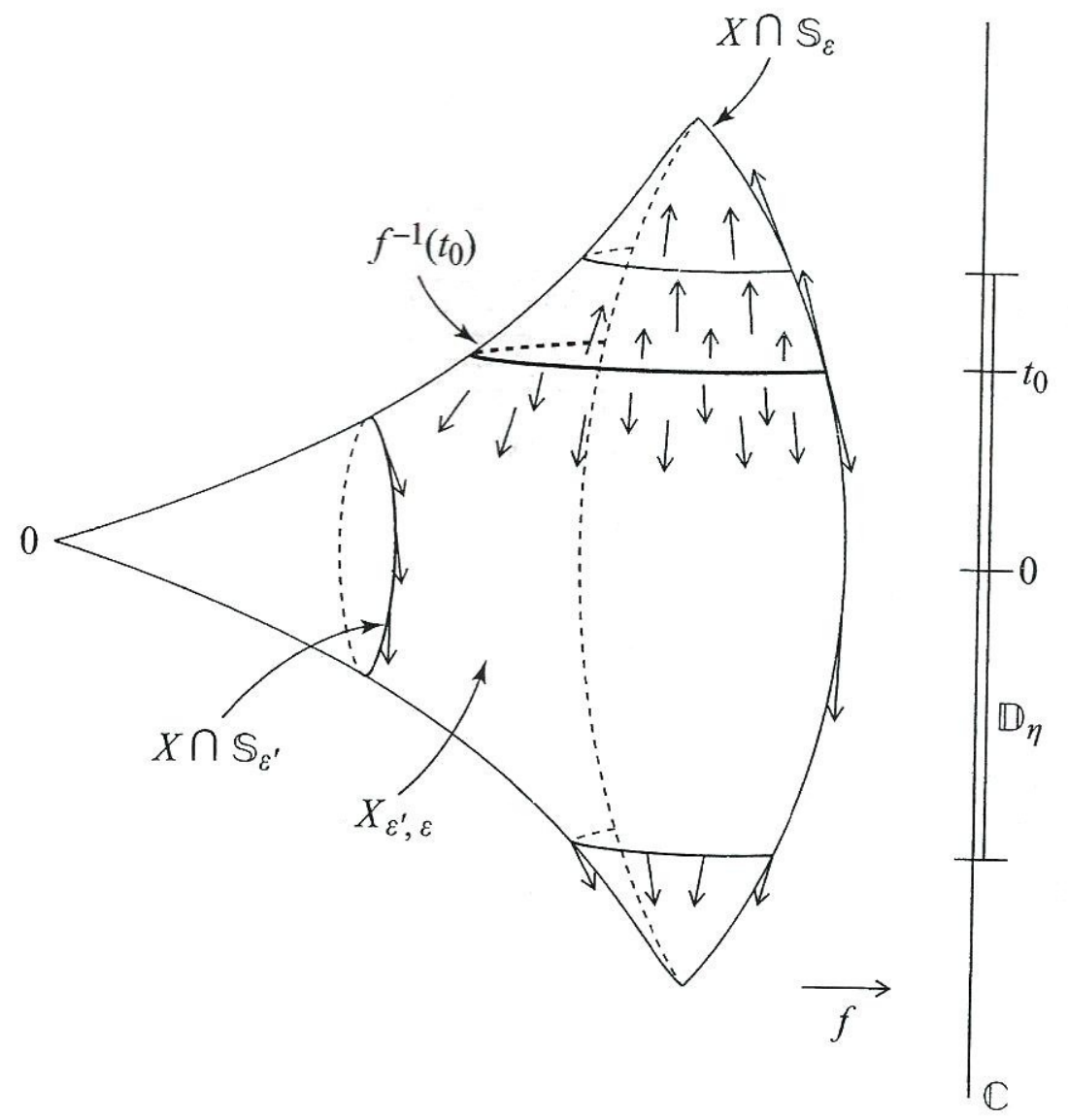

Demonstração. Por hipótese, a restrição de $f$ a todo estrato $V_{i}$ (exceto o estrato $\{0\}$ ) é regular, portanto $\operatorname{ker}(d f)$ tem codimensão 1 em $V_{i}$. 
A métrica Hermitiana em $\mathbb{C}^{n}$ induz uma métrica em $V_{i}$ e define uma divisão do fibrado tangente $T V_{i}$ como uma soma dos fibrados tangentes as fibras e o fibrado normal. A derivada $d f$ restrita ao fibrado normal é um isomorfismo.

Além disso, podemos levantar todo campo de vetores em $\mathbb{D}_{\eta}$ como um campo de vetores em $V_{i}$ e ortogonal as fibras $f^{-1}(t)$. Denotamos por $\xi$ o campo de vetores em $\mathbb{D}_{\eta}$, radial em $t_{0}$ e denotamos por $u_{i}$ o levantamento de $\xi$ em $V_{i}$.

Assim como na definição do campo de vetores $\bar{\nabla}_{X} f$, as condições de Whitney nos permite colar os campos $u_{i}$ formando um campo de vetores estratificado $u$ definido em $\left(\mathbb{B}_{\varepsilon} \cap f^{-1}\left(\mathbb{D}_{\eta}\right)\right) \backslash\{0\}$ e satisfazendo as condições do lema.

\subsection{Demonstração do Lema 3.1 .2}

Esta última seção é dedicada a demonstração do Lema 3.1.2, com o qual foi possível demonstrarmos o principal resultado apresentado neste trabalho.

Além dos Lemas 3.2.1 3.2.2 para a construção do campo de vetores $w$ do Lema 3.1 .2 utilizaremos também do resultado a seguir, cuja demonstração pode ser encontrada em [4].

Teorema 3.3.1 (Teorema 2.3 [4]). Seja $f$ uma função analítica definida em uma vizinhança aberta de 0 em $X \subset \mathbb{C}^{n}$, assuma $f(0)=0$. Então existe $\varepsilon_{0}>0$ tal que para todo $\varepsilon, 0<\varepsilon<\varepsilon_{0}$, existe $\eta_{\varepsilon}>0$ tal que se $v$ é um campo de vetores estratificado em $X \cap \mathbb{B}_{\varepsilon} \cap f^{-1}\left(\partial \mathbb{D}_{\eta}\right), 0<\eta<\eta_{\varepsilon}$, o qual é transversal à $X \cap \mathbb{S}_{\varepsilon}$ e à $X \cap f^{-1}\left(\partial \mathbb{D}_{\eta}\right)$ apontando para fora, então existe uma extensão estratificada de $v$ como um campo de vetores em $\left(X \cap \mathbb{B}_{\varepsilon}\right) \backslash f^{-1}\left(\operatorname{Int}\left(\mathbb{D}_{\eta}\right)\right)$, o qual é transversal à $X \cap \mathbb{S}_{\varepsilon}$ apontando para fora.

Demonstração do Lema 3.1.2. Primeiramente definiremos o campo de vetores $w$ em $\left(\mathbb{B}_{\varepsilon} \cap f^{-1}\left(\mathbb{D}_{\eta}\right)\right) \backslash \operatorname{Int}\left(\mathbb{B}_{\varepsilon^{\prime}}\right)$ como a soma (via partição da unidade) dos campos de vetores dados pelos Lemas 3.2.1 e 3.2.2. Obtemos um campo de vetores estratificado satisfazendo:

1. w é transversal à esfera $\mathbb{S}_{\varepsilon}$ apontando para fora;

2. $w$ é tangente à $Y_{t_{0}}$;

3. As singularidades de $w$ estão contidas em $Y_{t_{0}}$; 
4. Em cada estrato $Y_{t_{0}} \cap V_{i} \cap \mathbb{B}_{\varepsilon}, w$ somente se anula em um número finito de pontos e a soma dos índices de Poincaré-Hopf da restrição de $w$ à $Y_{t_{0}} \cap V_{i} \cap \mathbb{B}_{\varepsilon}$ é $\chi\left(Y_{t_{0}} \cap V_{i} \cap \mathbb{B}_{\varepsilon}\right)$;

5. Em cada ponto singular, $w$ é transversalmente radial ao estrato que contém o ponto singular;

6. $w$ é transversal à fronteira $f^{-1}\left(\partial \mathbb{D}_{\eta}\right)$ apontando para fora.

Então estendemos $w$ a todo $X_{\varepsilon^{\prime}, \varepsilon}$ usando o Teorema 3.3.1. Para completar a prova, note que restrito a $\mathbb{S}_{\varepsilon^{\prime}}$ o campo de vetores $w$ é homotópico ao campo de vetores gradiente $\bar{\nabla}_{X} f$. Este fato segue do Lema 2.5.3 e do fato que, em $\mathbb{S}_{\varepsilon^{\prime}}$, o campo de vetores $w$ é o campo de vetores $u$ do Lema 3.2 .2 , o qual é o levantamento de um campo de vetores homotópico à um campo de vetores constante próximo da origem.

Com a construção desse campo de vetores $w$, pudemos demonstrar o Teorema 3.1.1. cujo resultado nos apresenta uma relação existente entre a obstrução de Euler e a obstrução de Euler de uma função. 


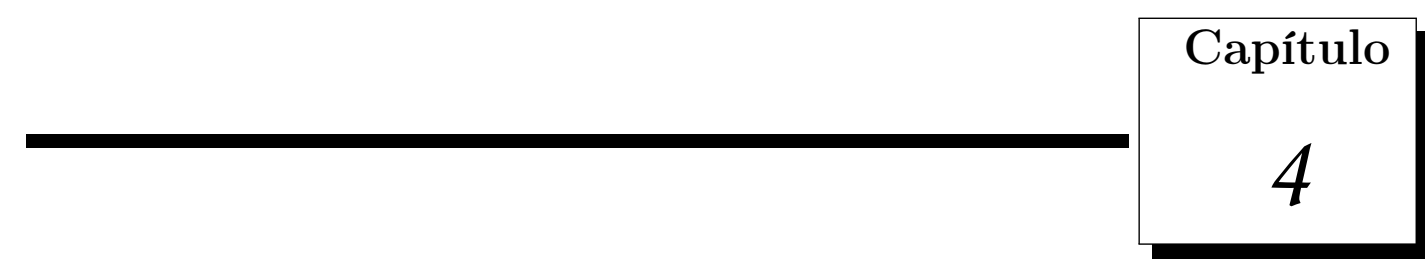

Conclusão

Neste trabalho, estudamos a obstrução de Euler definida para espaços analíticos complexos. Exibimos um importante resultado que nos permite calcular a obstrução de Euler a partir dos cálculos das obstruções de Euler dos estratos de uma estratificação de Whitney, assim como de suas características de Euler.

Apresentamos a definição de obstrução de Euler de uma função, para o caso de funções com singularidade isolada definidas em espaços analíticos complexos. Este conceito generaliza a obstrução de Euler e também generaliza um importante invariante da teoria de singularidades, o número de Milnor de uma função, tais relações foram verificadas e foram apresentados exemplos.

Uma observação importante é a comparação entre a obstrução de Euler de uma função $E u_{f, \mathbb{C}^{d}}(0)$, onde $f:\left(\mathbb{C}^{d}, 0\right) \rightarrow(\mathbb{C}, 0)$ é uma função analítica com singularidade isolada na origem e a obstrução de Euler $E u_{X}(0)$, onde $X=f^{-1}(0)$. Mais geralmente, observamos o caso em que $X$ é uma ICSI, neste caso, $E u_{X}(0)$ está relacionada ao número de Milnor de uma seção genérica de X.

Nosso principal resultado, o qual exibe uma relação entre a obstrução de Euler e a obstrução de Euler de uma função, foi amplamente estudado no capítulo 3, onde vários resultados técnicos foram provados, mostrando a relevância e complexidade deste resultado. 



\section{Referências Bibliográficas}

[1] J.-P. Brasselet, D. Massey, A. J. Parameswaran, and J. Seade. Euler obstruction and defects of functions on singular varieties. J. London Math. Soc. (2), 70(1):59-76, 2004.

[2] J.-P. Brasselet and M.-H. Schwartz. Sur les classes de Chern d'un ensemble analytique complexe. In The Euler-Poincaré characteristic (French), volume 82 of Astérisque, pages 93-147. Soc. Math. France, Paris, 1981.

[3] J.-P. Brasselet, J. Seade, and T. Suwa. Vector fields on singular varieties, volume 1987 of Lecture Notes in Mathematics. Springer-Verlag, Berlin, 2009.

[4] J.-P. Brasselet, Lê D. T., and J. Seade. Euler obstruction and indices of vector fields. Topology, 39(6):1193-1208, 2000.

[5] K. Burns and M. Gidea. Differential geometry and topology - with a view to dynamical systems. Studies in Advanced Mathematics. Chapman \& Hall/CRC, Boca Raton, FL, 2005.

[6] R. Callejas-Bedregal, M. J. Saia, and J. N. Tomazella. Euler obstruction and polar multiplicities of images of finite morphisms on ICIS. Proc. Amer. Math. Soc., 140(3):855-863, 2012.

[7] A. Douady. Variétés à bord anguleux et voisinages tubulaires. In Séminaire Henri Cartan, 1961/62, Exp. 1, page 11. Secrétariat mathématique, Paris, 1961/1962.

[8] C. G. Gibson. Singular points of smooth mappings, volume 25 of Research Notes in Mathematics. Pitman (Advanced Publishing Program), Boston, Mass., 1979.

[9] C. G. Gibson, K. Wirthmüller, A. A. du Plessis, and E. J. N. Looijenga. Topological stability of smooth mappings. Lecture Notes in Mathematics, Vol. 552. Springer-Verlag, Berlin, 1976.

[10] G. González-Sprinberg. L'obstruction locale d'Euler et le théorème de MacPherson. In The EulerPoincaré characteristic (French), volume 83 of Astérisque, pages 7-32. Soc. Math. France, Paris, 1981. 
[11] S. M. Guseum-Zade, I. Luengo, and A. Melle-Hernández. On the topology of germs of meromorphic functions and its applications. Algebra i Analiz, 11(5):92-99, 1999.

[12] H. Hamm. Lokale topologische Eigenschaften komplexer Räume. Math. Ann., 191:235-252, 1971.

[13] R. Hartshorne. Algebraic geometry. Graduate Texts in Mathematics, No. 52. Springer-Verlag, New York, 1977.

[14] E. L. Lima. Introdução à topologia diferencial. Notas de Matemática no 23, IMPA, 1961.

[15] E. L. Lima. Variedades diferenciáveis. Publicações matemáticas. IMPA, 2007.

[16] R. D. MacPherson. Chern classes for singular algebraic varieties. Ann. of Math. (2), 100:423-432, 1974.

[17] D. B. Massey. A little microlocal Morse theory. Math. Ann., 321(2):275-294, 2001.

[18] J. Milnor. Singular points of complex hypersurfaces. Annals of mathematics studies. Princeton University Press, 1968.

[19] J. W. Milnor. Topology from the differentiable viewpoint. Based on notes by D. W. Weaver. The University Press of Virginia, Charlottesville, Va., 1965.

[20] J. W. Milnor and J. D. Stasheff. Characteristic classes. Annals of Mathematics Studies, No. 76. Princeton University Press, Princeton, N. J., 1974.

[21] J. R. Munkres. Elements of algebraic topology. Addison-Wesley Publishing Company, Menlo Park, CA, 1984.

[22] M.-H. Schwartz. Champs radiaux sur une stratification analytique, volume 39 of Travaux en Cours [Works in Progress]. Hermann, Paris, 1991.

[23] M. Spivakovsky. Sandwiched singularities and desingularization of surfaces by normalized Nash transformations. Ann. of Math. (2), 131(3):411-491, 1990.

[24] Lê D. T. Le concept de singularité isolée de fonction analytique. In Complex analytic singularities, volume 8 of Adv. Stud. Pure Math., pages 215-227. North-Holland, Amsterdam, 1987.

[25] Lê D. T. Complex analytic functions with isolated singularities. J. Algebraic Geom., 1(1):83-99, 1992.

[26] F. Tari. Singularidades de aplicações diferenciáveis. São Carlos: Notas didáticas n 34, ICMC-USP, 1999.

[27] J. L. Verdier. Stratifications de Whitney et théorème de Bertini-Sard. Invent. Math., 36:295-312, 1976.

[28] H. Whitney. Tangents to an analytic variety. Ann. of Math. (2), 81:496-549, 1965. 